\title{
The Compactions of Elasto-Plastic and Visco-Plastic Granular Assemblies
}

\author{
Pengfei He, Yuching Wu, Huiliang Chen \\ College of Civil Engineering, Tongji University, Shanghai, China \\ Email: ycwu@tongji.edu.cn
}

Received November 16, 2012; revised December 18, 2012; accepted December 30, 2012

\begin{abstract}
In this paper, the compactions of the elasto-plastic and the visco-plastic granular assemblies are simulated using the finite element method. Governing equations for motion and deformation for particles, including coupling of rigid body motion and deformation for deformable bodies, are investigated. An implicit discrete element method for block systems is developed to make visco-plastic analysis for the assemblies. Among particles, three different contact types, cohering, rubbing and sliding, are taken into account. To verify accuracy and efficiency of the numerical method, some numerical example is simulated and the results are in a satisfactory agreement with the solutions in literatures. The effects of frictional condition, the initial solid volume ratio, the number of particles in the assembly, and different types of compacttion on the compaction of the elasto-plastic and the visco-plastic aggregates are investigated. It is demonstrated that the effect of frictional condition, the initial solid volume ratio, the number of particles in the assembly, and different types of compaction on the global behavior of the elasto-plastic the visco-plastic granular assemblies under compacting are considerable. The numerical model is extended to simulate the compaction of aggregates consisting of mixed particles of different viscous incompressible materials. It is indicated that, with minor modification, the method could be used in a variety of problems that can be represented using granular media, such as asphalt, polymers, aluminum, snow, food product, etc.
\end{abstract}

Keywords: Compaction; Elasto-Plastic; Visco-Plastic; Granular Assembly; Finite Element Method

\section{Introduction}

Researches focused on particulate materials were developed rapidly in the past 20 years. In the 1990s, basic physics of particle material characteristics were described in detail by Jaeger and Nagel [1], and fundamental theory of mechanics of granular materials was developed. At the same time, the load distribution for twodimensional aggregates compaction was studied by Luding [2]. For the issue of the particle shape, two-dimensional quasi-static analysis for circular particle compression state was made by Bashir and Goddard [3]. The frictional pressure of oval particles was taken into account in theoretical analysis and numerical simulation by Tzaferopoulos [4]. Coppersmith et al. [5] studied the problem of bead-like particles model. Matuttis [6] simulated twodimensional hexagonal particle under distributed loading. Satake [7] presented a random distribution method to define particle position based on theoretical and numerical analysis. Emeriault et al. [8] proposed a uniform method to create a structure consisting of the oval-shaped particles. Antony et al. [9] investigated limit state of compression and impact of aggregates consisting of the flat round, round and elongated spherical particles. It was demonstrated that the effect of particle shape on the final result was little. The same particle samples under different pressure distribution were studied. In the final state of compression of the particles, it was indicated that the shape and distribution of particles had little effect on the global elastic deformation resulted from rigid body movement of particles.

In addition, some scholars have begun to investigate compaction of particles of non-spherical shape. Wu [10] simulated compaction of adhesive polygonal particles in different size. A step-by-step incremental numerical model was developed to simulate the compaction of granular system of non-spherical nonlinear visco-plastic particles. The nonlinear visco-plastic constitutive law of each particle was derived based on the generalized Maxwell model that the material property of each element was treated as a series connection of a linear elastic matter to the other nonlinear visco-plastic media. The mesh upgrade scheme, consisting of contact detection algorithms, mesh update algorithms, concave four-node element modification, and locking prevention subroutine, was generated based on a mixed triangular and quadric- 
lateral mesh system [11,12]. However, these researches only focused on a perfectly plastic material and the use of particle equations for the nonlinear viscous process was not taken into account.

In this paper, uni-axial compaction of two-dimensional mixed non-linear viscous polygonal particles is examined. A numerical model is developed to anatomize the problem. It provides a theoretical basis and numerical model for engineering design related to particulate matter. This research mainly includes the following four sections. First of all, a non-linear numerical model is established to analyze amass made up of elastic-plastic and viscoplastic particles under densification. Secondly, This method is applied to analyze the relationship between stress and density of the metal powder or asphalt material during the process of compaction in different directions. Next, the effects of the distribution of particles, particle density throughout the structure, particle size and shape, and friction among particles on the stress distribution of the entire structure are inspected. Finally, the defects and limitations of the numerical model are examined to establish a better model.

\section{Geometrically Nonlinear Finite Element Formulations}

The incremental finite element method is usually used to solve geometrically nonlinear problems. The main purpose is to identify speed, displacement, stress, strain and other parameters of kinematics and static of objects in a series of discrete time points. Assume that all variables at time $t$ are known, all solutions of the problem can be obtained by solving all parameters at time $t+\Delta t$ and repeating the procedure. For the incremental method, and the principle of virtual work at time $t+\Delta t$ can be expressed as

$$
\begin{aligned}
{ }^{t+\Delta t} W= & \int_{t+\Delta t}{ }^{t+\Delta t}{ }^{t+\Delta t} t_{k} \delta u_{k}^{t+\Delta t} \mathrm{~d} S \\
& +\int_{t+\Delta t} \rho_{t+\Delta t}^{t+\Delta t} f_{k} \delta u_{k}^{t+\Delta t} \mathrm{~d} V,
\end{aligned}
$$

where ${ }^{t+\Delta t} W$ is the virtual work of the loading at time $t+$ $\Delta t . \delta u_{k}$ is virtual displacement at time $t+\Delta t .{ }_{t+\Delta t}^{t+\Delta t} f_{k}$ and ${ }_{t+\Delta t}^{t+\Delta t} t_{k}$ are body force and surface traction at time $t+\Delta t$, respectively. ${ }^{t+\Delta t} V,{ }^{t+\Delta t} S$ and ${ }^{t+\Delta t} \rho$ are volume area and density of the object at time $t+\Delta t$, respectively.

After linearization, the total Lagrangian formulation can be written as

$$
\begin{aligned}
& \int_{0_{V} 0} D_{i j k l 0} e_{k l} \delta_{0} e_{i j}{ }^{0} \mathrm{~d} V+\int_{0_{V}}{ }_{0}{ }^{t} S_{i j} \delta_{0} \eta_{i j}{ }^{0} \mathrm{~d} V, \\
& ={ }^{t+\Delta t} W-\int_{0_{V}}{ }_{0}{ }^{t} S_{i j} \delta_{0} e_{i j}{ }^{0} \mathrm{~d} V
\end{aligned}
$$

The updated Lagrangian formulation can be written as

$$
\begin{aligned}
& \int_{{ }^{t} V}{ }_{t} D_{i j k l t} e_{k l} \delta_{t} e_{i j}{ }^{t} \mathrm{~d} V+\int_{{ }^{t} V}{ }^{t} \tau_{i j} \delta_{t} \eta_{i j}{ }^{t} \mathrm{~d} V \\
& ={ }^{t+\Delta t} W-\int_{{ }^{t} V}{ }^{t} \tau_{i j} \delta_{t} e_{i j}{ }^{t} \mathrm{~d} V,
\end{aligned}
$$

where $\delta_{t+\Delta t} e_{i j}$ is the corresponding infinitesimal strain written as

$$
\delta_{t+\Delta t} e_{i j}=\delta \frac{1}{2}\left({ }_{t+\Delta t} u_{i, j}+{ }_{t+\Delta t} u_{j, i}\right) .
$$

If the isoparametric element is used for solving the discrete domain, the coordinates of each element and the displacement of its nodes can be interpolated as follows.

$$
\begin{aligned}
& { }^{0} x_{i}=\sum_{k=1}^{n} N_{k}{ }^{0} x_{i}^{k},{ }^{t} x_{i}=\sum_{k=1}^{n} N_{k}{ }^{t} x_{i}^{k}, \\
& { }^{t+\Delta t} x_{i}=\sum_{k=1}^{n} N_{k}{ }^{t+\Delta t} x_{i}^{k},(i=1,2,3) \\
& { }^{t} u_{i}=\sum_{k=1}^{n} N_{k}{ }^{t} u_{i}^{k}, u_{i}=\sum_{k=1}^{n} N_{k} u_{i}^{k},(i=1,2,3),
\end{aligned}
$$

where ${ }^{t} x_{i}^{k}$ is the component of node $k$ at time $t$ in $i$ direction, ${ }^{t} u_{i}^{k}$ is the displacement component of node $k$ at time $t$ in $i$ direction. $N_{k}$ is the shape function of node $k$. $n$ is the number of nodes.

Substitution of Equations (3) and (4) to Equation (2) yields the total Lagrangian formulation given as

$$
\left({ }_{0}^{t} K_{L}+{ }_{0}^{t} K_{N L}\right) u={ }^{t+\Delta t} Q-{ }_{0}^{t} F,
$$

where $u$ is nodal incremental displacement vector. ${ }_{0}^{t} K_{L},{ }_{0}^{t} K_{N L}$ and ${ }_{0}^{t} F$ can be expressed as

$$
\begin{aligned}
& { }_{0}^{t} K_{L}=\sum_{e} \int_{0_{V_{e}}}{ }_{0}^{t} B_{L 0}^{T} D_{0}^{t} B_{L}{ }^{0} \mathrm{~d} V, \\
& { }_{0}^{t} K_{N L}=\sum_{e} \int_{0_{V_{e}}}{ }_{0}^{t} B_{N L}^{T}{ }_{0}^{t} S_{0}^{t} B_{N L}{ }^{0} \mathrm{~d} V, \\
& { }_{0}^{t} F=\sum_{e} \int_{{ }_{V_{e}}}{ }_{0}^{t} B_{L}^{T}{ }_{0}^{t} \hat{S}^{0} \mathrm{~d} V,
\end{aligned}
$$

here ${ }_{0}^{t} B_{L}$ and ${ }_{0}^{t} B_{N L}$ are the transformation matrix of linear strain ${ }_{0} e_{i j}$ and nonlinear strain ${ }_{0} \eta_{i j}$, respectively. ${ }_{0} D$ is the constitutive matrix. ${ }_{0}^{t} S$ and ${ }_{0}^{t} \hat{S}$ is the second Piola-Kirchhoff stress matrix and vector, respectively. All of these parameters are measured from the initial configuration corresponding to the shape and position at time $t$.

Using the modified Newton-Raphson iteration, sufficiently accurate solution of Equation (9) can be obtained. That is

$$
\left({ }_{0}^{t} K_{L}+{ }_{0}^{t} K_{N L}\right) \Delta u^{(l)}={ }^{t+\Delta t} Q-{ }_{0}^{t+\Delta t} F^{(l)} \quad(l=0,1,2, \cdots),
$$

where

$$
\begin{aligned}
& { }^{t+\Delta t} u^{(l+1)}={ }^{t+\Delta t} u^{(l)}+\Delta u^{(l)}, \\
& { }^{t+\Delta t} F^{(l)}=\int_{0_{V}}{ }^{t+\Delta t}{ }_{0}^{(l)} B_{L}^{(l)}{ }^{t+\Delta t} \hat{S}^{(l) 0} \mathrm{~d} V .
\end{aligned}
$$

Similarly, subsititution of Equations (6)-(8) to Equation (3) yields the updated Lagrangian formulation given as 


$$
\left({ }_{t}^{t} K_{L}+{ }_{t}^{t} K_{N L}\right) u={ }^{t+\Delta t} Q-{ }_{t}^{t} F,
$$

where

$$
\begin{aligned}
& { }_{t}^{t} K_{L}=\sum_{e} \int_{{ }^{t} V_{e}}{ }_{t}^{t} B_{L t}^{T} D_{t}^{t} B_{L}{ }^{t} \mathrm{~d} V, \\
& { }_{t}^{t} K_{N L}=\sum_{e} \int_{t^{t}{ }_{e}}{ }_{t}^{t} B_{N L}^{T}{ }^{t} \tau_{t}^{t} B_{N L}{ }^{t} \mathrm{~d} V, \\
& { }_{t}^{t} F=\sum_{e} \int_{0_{V_{e}}{ }_{t}{ }_{t}^{t} B_{L}^{T}{ }^{T} \hat{\tau}^{t} \mathrm{~d} V,}
\end{aligned}
$$

here ${ }_{t}^{t} B_{L}$ and ${ }_{t}^{t} B_{N L}$ are transformation matrix of linear strain ${ }_{t} e_{i j}$ and nonlinear strain ${ }_{t} \eta_{i j}$, respectively. ${ }_{t} D$ is constitutive matrix. ${ }^{t} \tau$ and ${ }^{t} \hat{\tau}$ are Cauchy stress matrix and vector, respectively. All of these parameters are measured from the current configuration corresponding to the shape and position at time $t$.

Using the modified Newton-Raphson iteration, sufficiently accurate solution of Equation (16) can be obtained. That is

$$
\begin{aligned}
& \left({ }_{t}^{t} K_{L}+{ }_{t}^{t} K_{N L}\right) \Delta u^{(l)} \\
& ={ }^{t+\Delta t} Q-{ }_{t+\Delta t}^{t+\Delta t} F^{(l)}(l=0,1,2, \cdots)
\end{aligned}
$$

where

$$
{ }_{t+\Delta t}^{t+\Delta t} F^{(l)}=\int_{t+\Delta t}{ }^{t+\Delta t}{ }^{t+\Delta t} B_{L}^{(l) T}{ }^{t+\Delta t} \hat{\tau}^{(l)}{ }^{t+\Delta t} \mathrm{~d} V^{(l)} .
$$

\section{Finite Element Methods for Contact Problems}

In the contact problems, the interfacial conditions between two objects can be classified into two types, touch and separation. And the situations of touch can also be classified into two types, sticking and sliding. Assuming the object $A$ and the object $B$ as the two domains, the equivalent principle of virtual displacement can be expressed as

$$
\begin{aligned}
& \int^{t+\Delta t_{V}}{ }^{t+\Delta t} \tau_{i j} \delta_{t+\Delta t} e_{i j}{ }^{t+\Delta t} \mathrm{~d} V-{ }^{t+\Delta t} W_{L}-{ }^{t+\Delta t} W_{I}-{ }^{t+\Delta t} W_{c} \\
& =\sum_{r=}^{A, B}\left[\int^{t+\Delta t} V^{r}{ }^{t+\Delta t} \tau_{i j}^{r} \delta{ }^{t+\Delta t} e_{i j}^{r}{ }^{r+\Delta t} \mathrm{~d} V\right. \\
& \left.-{ }^{t+\Delta t} W_{L}^{r}-{ }^{t+\Delta t} W_{I}^{r}-{ }^{t+\Delta t} W_{c}^{r}\right]=0
\end{aligned}
$$

where ${ }^{t+\Delta t} W_{L},{ }^{t+\Delta t} W_{I}$ and ${ }^{t+\Delta t} W_{c}$ are the virtual work of external forces, inertial forces and contact forces loaded on the configuration at time $t+\Delta t$, respectively.

If there is friction between two contact surfaces, the virtual work is expressed as

$$
\begin{aligned}
& { }^{t+\Delta t} W_{c}=-\delta \Pi_{C P}=\int_{{ }^{t+\Delta t} S_{c}}-\alpha_{N}\left(u_{N}^{A}-u_{N}^{B}+{ }^{t} \bar{g}_{N}\right) \\
& \times\left[\left(\delta u_{N}^{A}-\delta u_{N}^{B}\right)-\mu\left(\bar{u}_{J} / \bar{u}_{T}\right) \cdot\left(\delta u_{J}^{A}-\delta u_{J}^{B}\right)\right]^{t+\Delta t} \mathrm{~d} S \\
& \quad(J=1,2)
\end{aligned}
$$

For the situation without friction, we can let $\mu=0$ to get the corresponding equation.

Next, the corresponding finite element models are generated as follows. The total Lagrangian formulation in matrix form can be given as

$$
M^{t+\Delta t} \ddot{u}+\left({ }_{0}^{t} K_{L}+{ }_{0}^{t} K_{N L}\right) u-{ }^{t+\Delta t} Q_{c}={ }^{t+\Delta t} Q_{L}-{ }_{0}^{t} F,
$$

and the total Lagrangian formulation in matrix form can be given as

$$
M^{t+\Delta t} \ddot{u}+\left({ }_{t}^{t} K_{L}+{ }_{t}^{t} K_{N L}\right) u-{ }^{t+\Delta t} Q_{c}={ }^{t+\Delta t} Q_{L}-{ }_{t}^{t} F,
$$

where $Q_{L}$ is equivalent nodal loading vector.

For sticking situation, the Lagrangian multiplier model can be written in T.L. form as

$$
\begin{aligned}
& M^{t+\Delta t} \ddot{u}+\left[\begin{array}{cc}
{ }_{0}^{t} K_{L}+{ }_{0}^{t} K_{N L} & K_{c \lambda} \\
K_{c \lambda}^{T} & 0
\end{array}\right]\left(\begin{array}{c}
u \\
t+\Delta t \lambda
\end{array}\right) \\
& =\left(\begin{array}{c}
{ }^{t+\Delta t} Q_{L}-{ }_{0}^{t} F \\
-{ }^{t} \bar{g}
\end{array}\right)
\end{aligned}
$$

in U.L. form,

$$
\begin{aligned}
& M^{t+\Delta t} \ddot{u}+\left[\begin{array}{cc}
{ }_{t}^{t} K_{L}+{ }_{t}^{t} K_{N L} & K_{c \lambda} \\
K_{c \lambda}^{T} & 0
\end{array}\right]\left(\begin{array}{c}
u \\
t+\Delta t \lambda
\end{array}\right) \\
& =\left(\begin{array}{c}
{ }^{t+\Delta t} Q_{L}-{ }_{t}^{t} F \\
-{ }^{t} \bar{g}
\end{array}\right)
\end{aligned}
$$

For sliding situation, the Lagrangian multiplier model can be written in T.L. form as

$$
\begin{aligned}
& M^{t+\Delta t} \ddot{u}+\left[\begin{array}{cc}
{ }_{0}^{t} K_{L}+{ }_{0}^{t} K_{N L} & K_{c \lambda} \\
K_{c u} & 0
\end{array}\right]\left(\begin{array}{c}
u \\
{ }^{t+\Delta t} \lambda_{N}
\end{array}\right) \\
& =\left(\begin{array}{c}
{ }^{t+\Delta t} Q_{L}-{ }_{0}^{t} F \\
-{ }^{t} \bar{g}_{N}
\end{array}\right),
\end{aligned}
$$

in U.L. form,

$$
\begin{aligned}
& M^{t+\Delta t} \ddot{u}+\left[\begin{array}{cc}
{ }_{t}^{t} K_{L}+{ }_{t}^{t} K_{N L} & K_{c \lambda} \\
K_{c u} & 0
\end{array}\right]\left(\begin{array}{c}
u \\
t+\Delta t \\
{ }^{t} \lambda_{N}
\end{array}\right) \\
& =\left(\begin{array}{c}
{ }^{t+\Delta t} Q_{L}-{ }_{t}^{t} F \\
-{ }^{t} \bar{g}_{N}
\end{array}\right) .
\end{aligned}
$$

Similarly, using the penalty method, the corresponding finite element formulation in matrix form can be written in T.L. form as

$$
\begin{aligned}
& M^{t+\Delta t} \ddot{u}+\left({ }_{0}^{t} K_{L}+{ }_{0}^{t} K_{N L}+K_{c \alpha}\right) u \\
& ={ }^{t+\Delta t} Q_{L}+{ }^{t+\Delta t} Q_{c}-{ }_{0}^{t} F,
\end{aligned}
$$

in U.L. form,

$$
\begin{aligned}
& M^{t+\Delta t} \ddot{u}+\left({ }_{t}^{t} K_{L}+{ }_{t}^{t} K_{N L}+K_{c \alpha}\right) u \\
& ={ }^{t+\Delta t} Q_{L}+{ }^{t+\Delta t} Q_{c}-{ }_{t}^{t} F,
\end{aligned}
$$


where

$$
\begin{aligned}
& K_{c \alpha}=\sum_{k=1}^{n_{c}}\left(K_{c \alpha}\right)_{k}, u_{c}=\sum_{k=1}^{n_{c}}\left(u_{c}\right)_{k}, \\
& { }^{t+\Delta t} \tilde{Q}_{c}=\sum_{k=1}^{n_{c}}\left({ }^{t+\Delta t} \tilde{Q}_{c}\right)_{k} .
\end{aligned}
$$

\section{Verification}

Compaction of the rigid elliptical particles has been simulated by Tzaferopoulos [4]. In this section, one of his numerical examples is simulated using the present numerical model. Results of both models are in a satisfactory agreement.

The finite element mesh of the numerical example is presented. Since in the original literature particles are set to be of rigid body. The elastic modulus in the numerical model is set as $2 \times 10^{10} \mathrm{MPa}$, and Poisson's ratio 0.5. At the same time, the friction coefficient of contacts among 25 particles is set to be 0.1 . Relation of particles to the surrounding rigid surface is set as contact state. The boundaries on the side and the bottom are fixed. The boundary on the top goes down in uniform velocity until it can not continue going down.

Final configurations from the present numerical model and literature are shown in Figure 1. It is indicated that final positions of particles are a little bit different between two results. However, layout and location of particles are very close to literature especially on the circled areas. It is demonstrated that the present numerical model is accurate and efficient.

\section{Numerical Experiments}

In this section, first of all, elasto-plastic analyses of aluminum granular materials are made using the proposed numerical model to get some properties of behavior of the aggregates in the compaction process. Next, viscoplastic analyses of asphalt aggregates are made to investtigate the global constitutive law. Then, mixed visco-

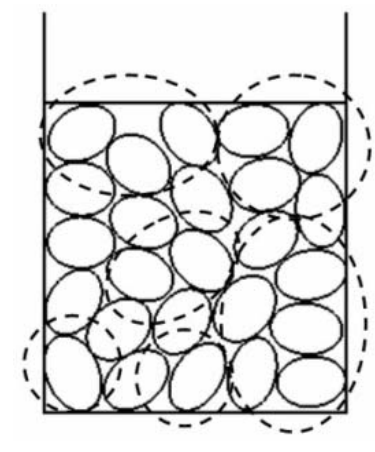

(a)

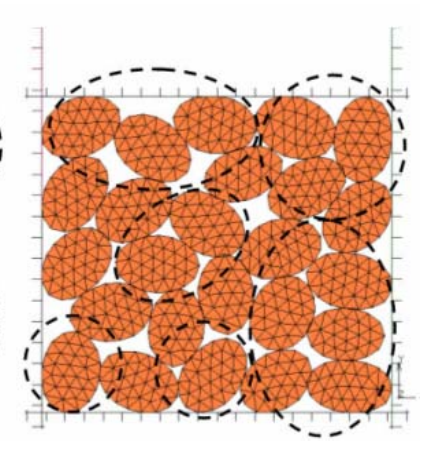

(b)
Figure 1. Final configurations from (a) literature and (b) the present numerical model. plastic analyses for assembles of three types of asphalt are made to offer some reference data for asphalt Industry.

\subsection{The Elasto-Plastic Analysis}

A series of elasto-plastic analyses of aluminum granular materials are made using the proposed numerical model to get some rules in the compaction process. The linear hardening elastic-plastic model is used as material model in the cases of temperature from $20^{\circ} \mathrm{C}$ to $250^{\circ} \mathrm{C}$. It can be simplified to the ideal elastic-plastic model at temperature higher than $250^{\circ} \mathrm{C}$. The stress-strain relation of aluminum alloy at $250^{\circ} \mathrm{C}$ is given in Table $\mathbf{1}$. In the elastic section elastic modulus is $64.1 \mathrm{GPa}$, and Poisson's ratio 0.3 .

The compaction for two-dimensional assembly of 20 particles is simulated. The particles are placed on the border of 1 meter square in area. The initial grain boundary area is around $65 \%$ of the total area. The simulation is regarded as a plane strain calculation. The compactions of two-dimensional aggregates of twenty particles can be classified as three types, the lateral compaction, the vertical compaction, and the bi-axial compaction. Friction between particles and wall is neglected. The inward movement of walls is of $10 \mathrm{~mm} / \mathrm{s}$ in velocity. Relationship of the wall pressure to solid ratio is obtained.

\subsubsection{The Lateral Compaction}

In this simulation, the total loading time is set to be $40 \mathrm{~s}$, with a total of 130 time steps. In the 124th step, the assembly is compacted to $98.43 \%$ of the initial area. The compaction process is shown in Figure 2.

Figure 3 shows the relationship between the wall pressure and the solid ratio. The value of lateral pressure begins to increase when the solid ratio is about 0.77. A large increase in slope occurs when the solid ratio is close to 1 . When the solid ratio is $98.43 \%$, the average values of the left and right wall pressure is $290 \mathrm{MPa}$, and the one of the top and bottom wall pressure 224.8 MPa. For the same solid ratio, the left-right pressure is less than the up-down pressure. At the same time, it is shown that the curve is oscillated rather than smooth. It is resulted from the complex interaction among the particles.

\subsubsection{The Vertical Compaction}

In this simulation, the total loading time is set to be $50 \mathrm{~s}$, with a total of 170 time steps. In the 128th step, the assembly is compacted to $97.90 \%$ of the initial area. The

Table 1. The stress-strain relation of aluminum alloy at $250^{\circ} \mathrm{C}$.

\begin{tabular}{llcccc}
\hline Strain (\%) & 0 & 0.11 & 0.31 & 0.87 & 1.60 \\
\hline Stress (MPa) & 0 & 70.5 & 111.2 & 129.1 & 130.2 \\
\hline
\end{tabular}




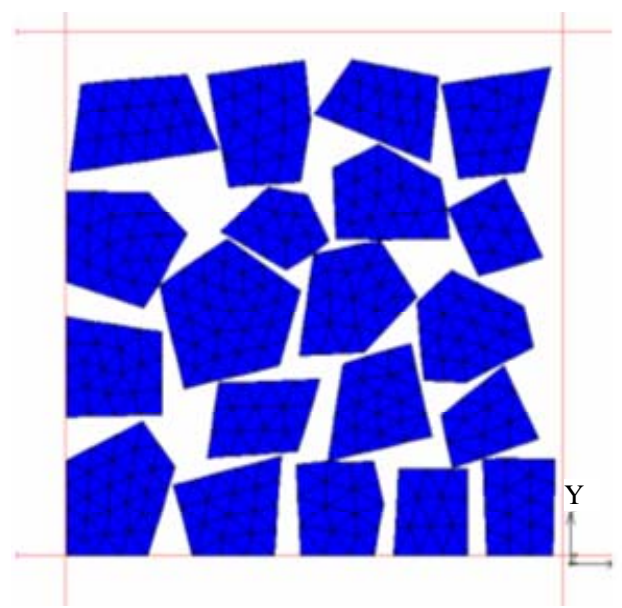

(a)

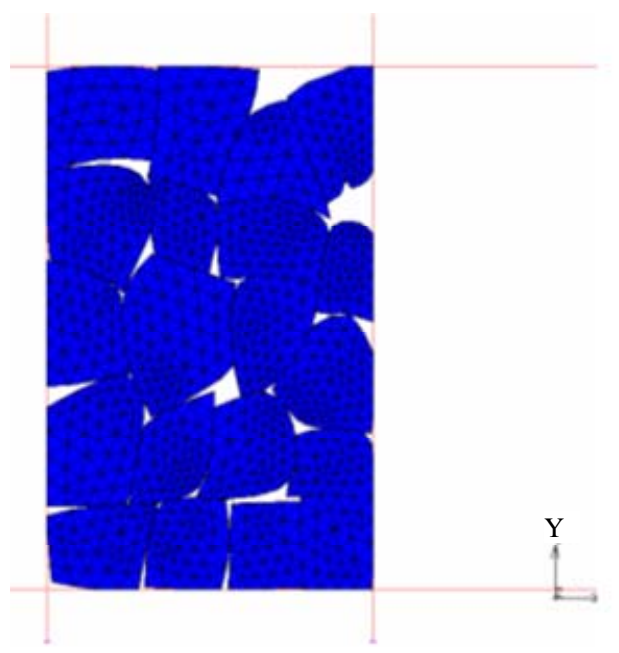

(c)

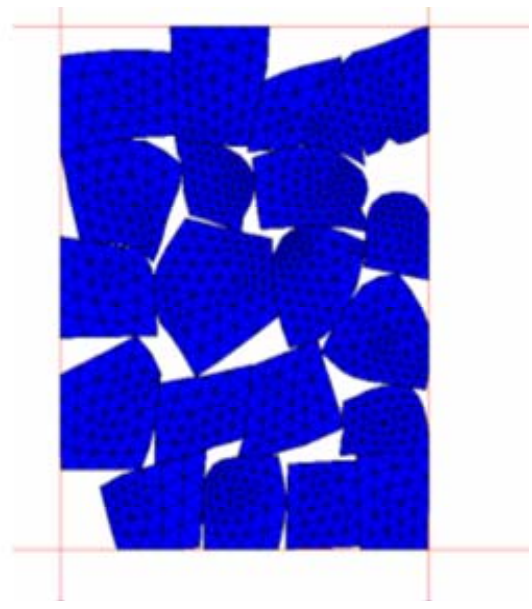

(b)

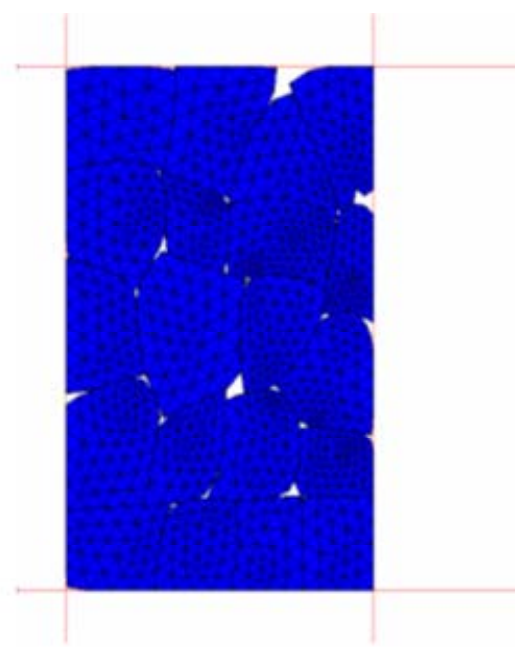

(d)

Figure 2. The process of the lateral compaction at: (a) The first time step; (b) The 85th time step; (c) The 113th time step; (d) The 124th time step.

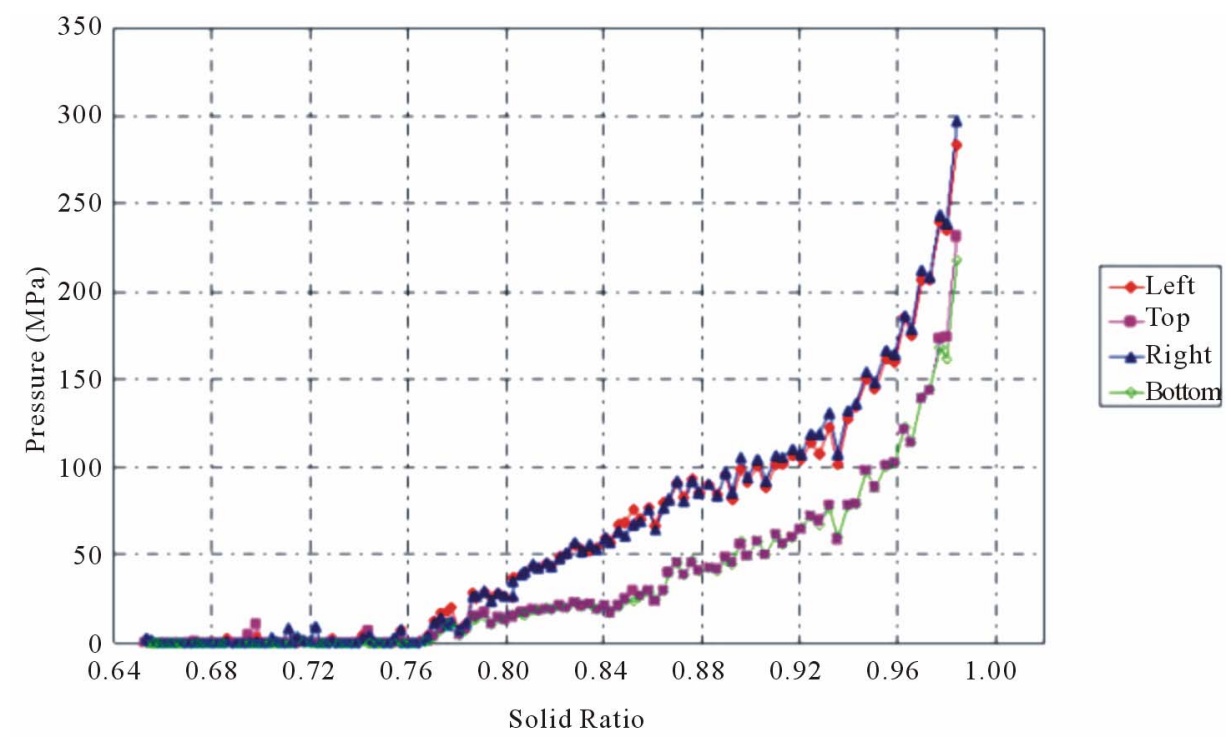

Figure 3. The relationship between the wall pressure and the solid ratio. 
compaction process is shown in Figure 4.

Figure 5 shows the relationship between the wall pressure and the solid ratio. The value of lateral pressure begins to increase when the solid ratio is about 0.79. A large increase in slope occurs when the solid ratio is close to 1 . When the solid ratio is $97.9 \%$, the average values of the left and right wall pressure is $290.8 \mathrm{MPa}$, and the one of the top and bottom wall pressure 228.9 $\mathrm{MPa}$. For the same solid ratio, the left-right pressure is less than the up-down pressure. At the same time, it is shown that the curve is oscillated rather than smooth. It is resulted from the complex interaction among the particles.

\subsubsection{The Biaxial Compaction}

In this simulation, the total loading time is set to be $40 \mathrm{~s}$, with a total of 130 time steps. In the 89th step, the assembly is compacted to $99.37 \%$ of the initial area. The compaction process is shown in Figure 6.

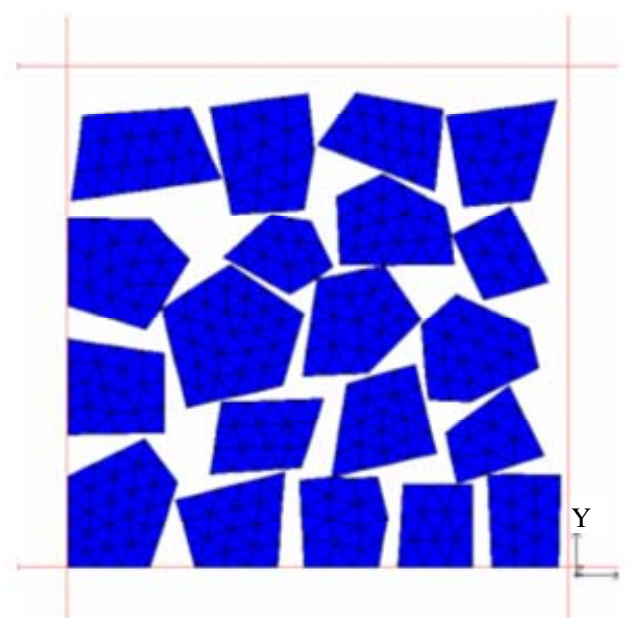

(a)

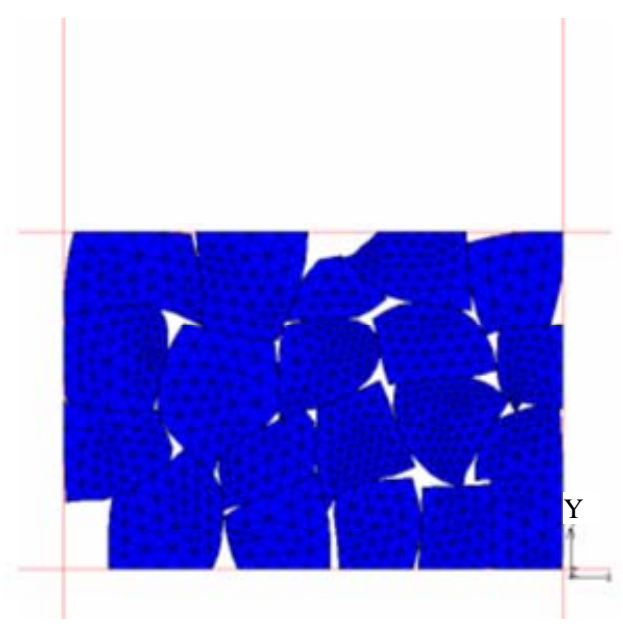

(c)
Figure 7 shows the relationship between the wall pressure and the solid ratio. The value of lateral pressure begins to increase when the solid ratio is about 0.79 . A large increase in slope occurs when the solid ratio is close to 1 . When the solid ratio is $99.37 \%$, the values of the left wall pressure is $290.9 \mathrm{Mpa}$, the one of the right wall pressure is $309.9 \mathrm{MPa}$, the one of the top wall pressure is $280.3 \mathrm{MPa}$, and the one of the bottom wall pressure is 297.2 MPa. For the same solid ratio, the left-right pressure is less than the up-down pressure. At the same time, it is shown that the curve is oscillated rather than smooth. It is resulted from the complex interaction among the particles.

\subsection{The Visco-Plastic Analysis}

In this section, a series of visco-plastic analyses of asphalt aggregates are made to investigate the global constitutive law. Here asphalt is used as the granular matter.

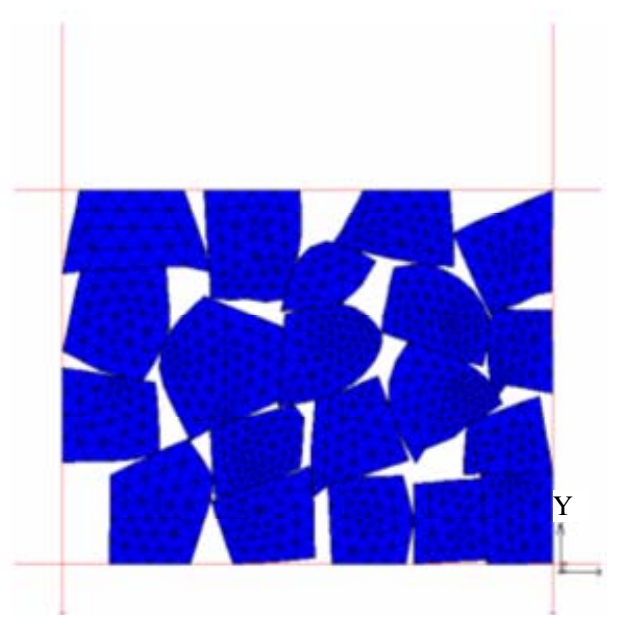

(b)

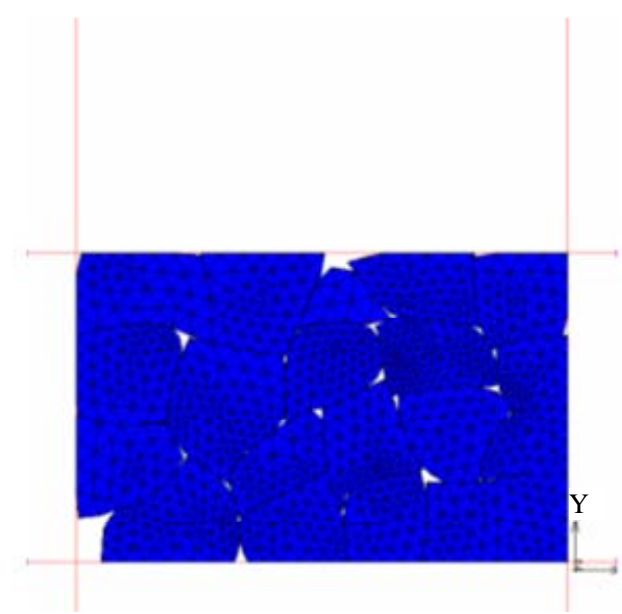

(d)

Figure 4. The process of the vertical compaction at: (a) The first time step; (b) The 82nd time step; (c) The 113th time step; (d) The 128th time step. 


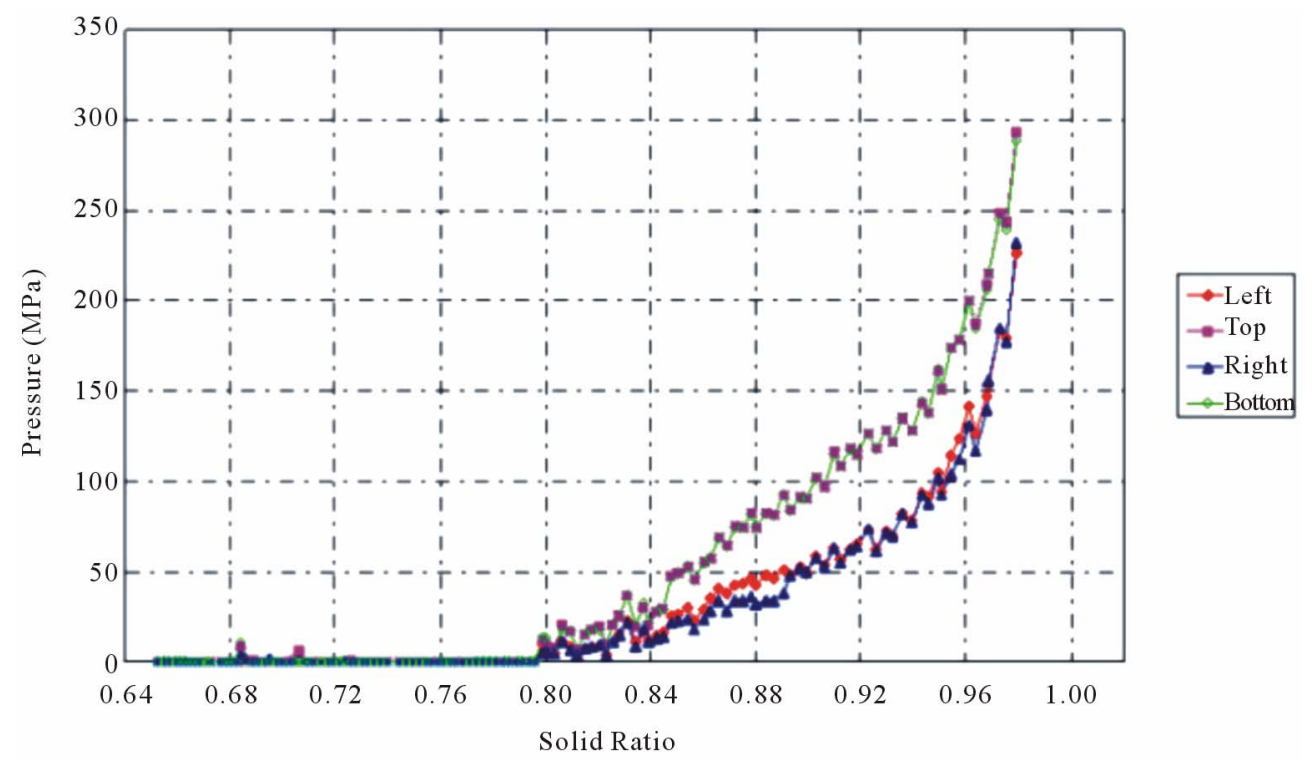

Figure 5. The relationship between the wall pressure and the solid ratio.

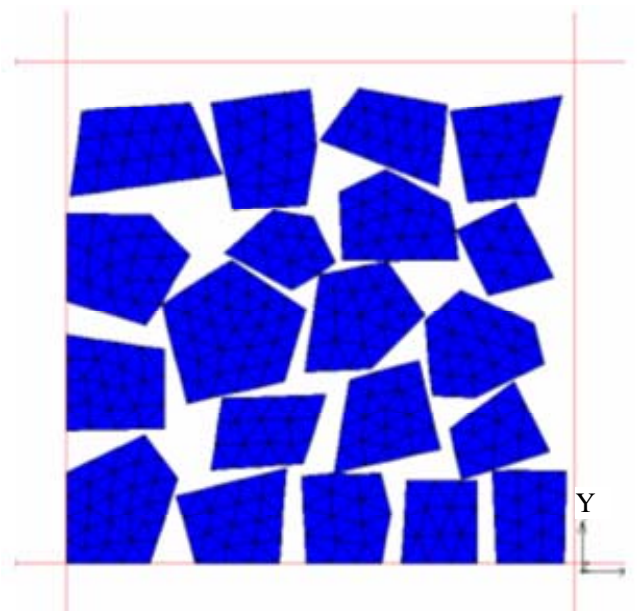

(a)

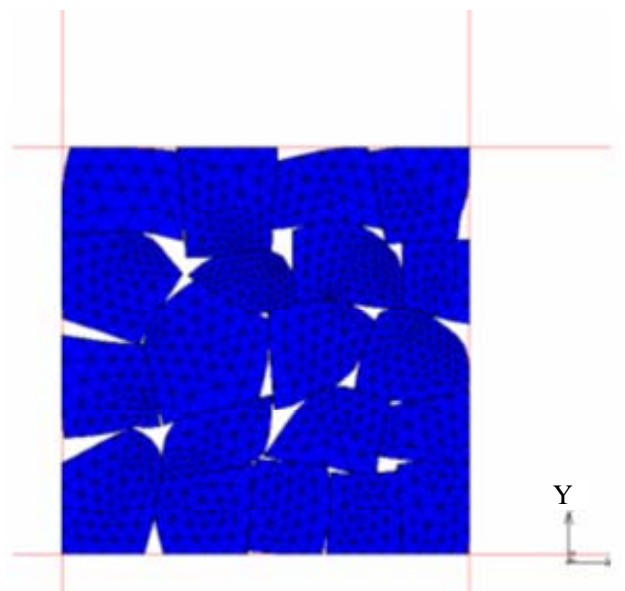

(c)

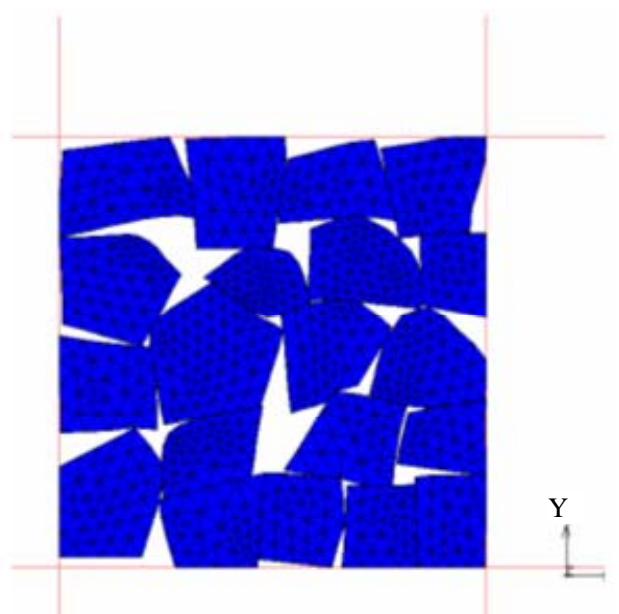

(b)

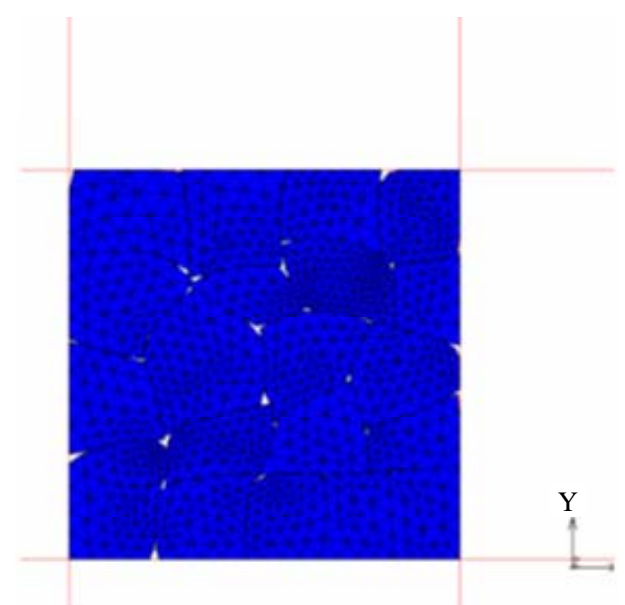

(d)

Figure 6. The process of the vertical compaction at: (a) The first time step; (b) The 61st time step; (c) The 78th time step; (d) The 89th time step. 


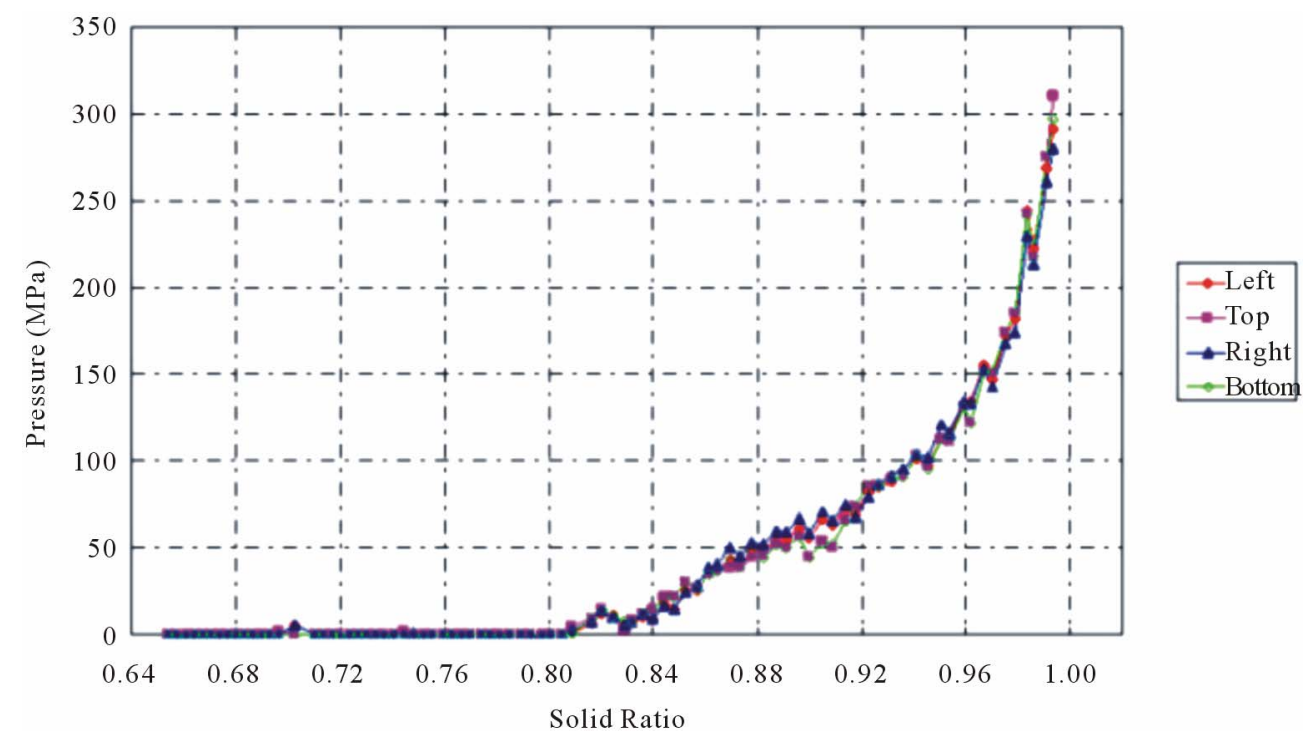

Figure 7. The relationship between the wall pressure and the solid ratio.

For the asphalt mixture, the visco-plastic strain is accumulated under cyclic loading. Generally, it is difficult to distinguish between visco-elastic and visco-plastic parts. And visc-oelastic properties can be changed under cyclic loading. Creep model is given as

$$
\dot{\varepsilon}_{c}=A \sigma^{n} \varepsilon^{c m} T^{p}\left(q t^{q-1}\right) .
$$

Parameters of the asphalt material properties are listed in Table 2.

Compaction for two-dimensional assembly of 16 particles is simulated. The particles are placed on the border of 1 meter square in area. The initial grain boundary area is around $80 \%$ of the total area. The simulation is regarded as a plane strain calculation. The compactions of two-dimensional aggregates of 16 particles can be classified as three types, the lateral compaction, the vertical compaction, and the two-way compaction. Large deflecttion and large strain are taken into account in the process of the compaction. The large deflection and large strain operators are set in the calculation here. Meanwhile, to ensure the convergence of computation, all deformed meshes are generated adaptively. Friction between particles and wall is neglected. The inward movement of walls is of $10 \mathrm{~mm} / \mathrm{s}$ in velocity. Relationship of the wall pressure to solid ratio is obtained.

\subsubsection{The Lateral Compaction}

In this simulation, the total loading time is set to be $43 \mathrm{~s}$, with a total of 118 time steps. In the 55th step, the assembly is compacted to $99.13 \%$ of the initial area. The compaction process is shown in Figure 8.

Figure 9 shows the relationship between the wall pressure and the solid ratio. The value of lateral pressure begins to increase when the solid ratio is about 0.86 . A
Table 2. Parameters of the asphalt material properties.

\begin{tabular}{cccccccc}
\hline \multicolumn{3}{c}{ Creep } & \multicolumn{4}{c}{ Elastic } \\
\hline$A$ & $m$ & $n$ & $p$ & $q$ & $E / \mathrm{MPa}$ & $\mu$ \\
$05.15 \times 10^{-8}$ & 0 & 0.826 & 0 & 0.22 & 526 & 0.5 \\
\hline
\end{tabular}

large increase in slope occurs when the solid ratio is close to 1 . When the solid ratio is $99.13 \%$, the average values of the left and right wall pressure is $128.4 \mathrm{MPa}$, and the one of the top and bottom wall pressure 62.5 MPa. For the same solid ratio, the left-right pressure is less than the up-down pressure.

\subsubsection{The Vertical Compaction}

In this simulation, the total loading time is set to be $43 \mathrm{~s}$, with a total of 118 time steps. In the 55th step, the assembly is compacted to $99.19 \%$ of the initial area. The compaction process is shown in Figure 10.

Figure 11 shows the relationship between the wall pressure and the solid ratio. The value of lateral pressure begins to increase when the solid ratio is about 0.86 . A large increase in slope occurs when the solid ratio is close to 1 . When the solid ratio is $99.19 \%$, the average values of the left and right wall pressure is 162.7.4 MPa, and the one of the top and bottom wall pressure 97.5 $\mathrm{MPa}$. For the same solid ratio, the left-right pressure is less than the up-down pressure.

\subsubsection{The Biaxial Compaction}

In this simulation, the total loading time is set to be $43 \mathrm{~s}$, with a total of 197 time steps. In the 49th step, the assembly is compacted to $99.28 \%$ of the initial area. The compaction process is shown in Figure 12. 


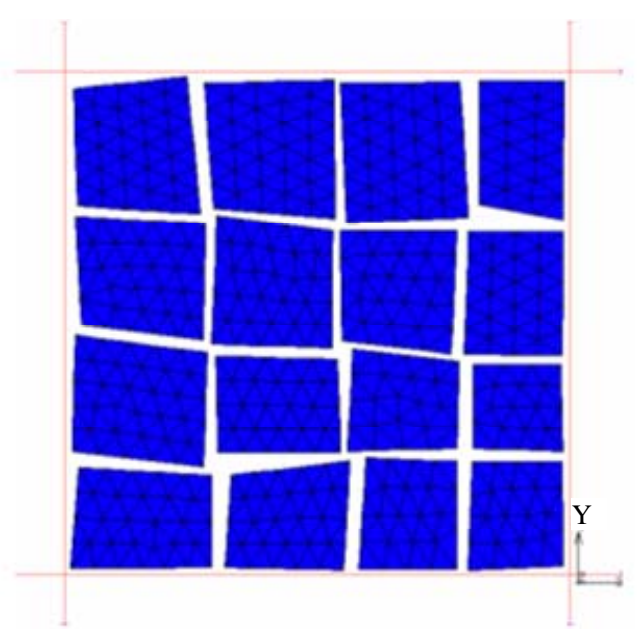

(a)

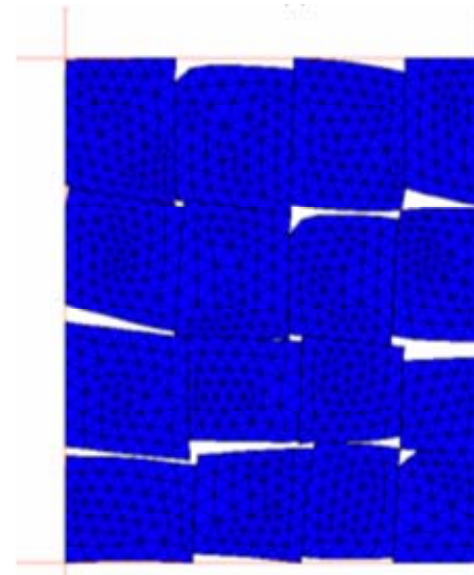

(c)

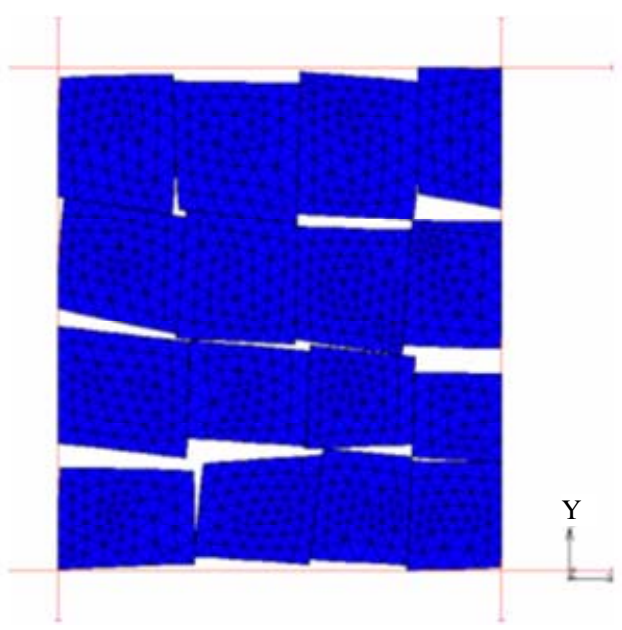

(b)

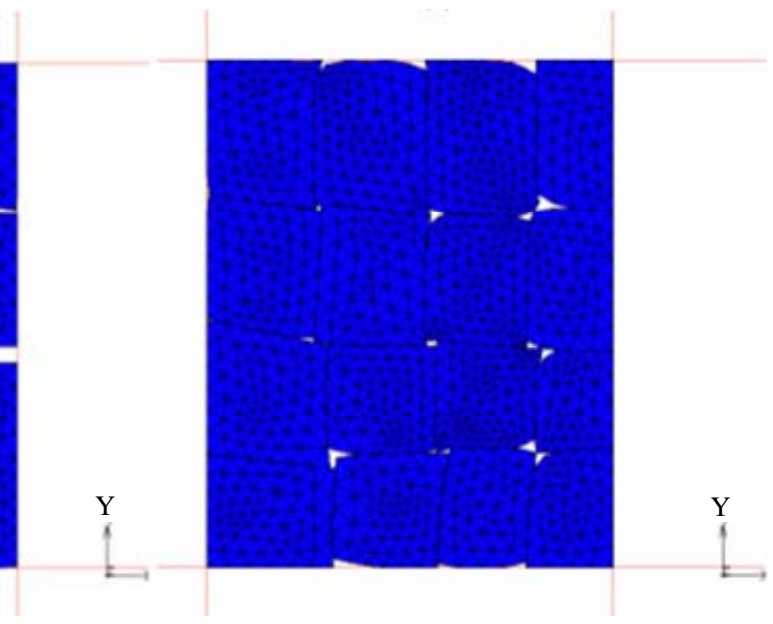

(d)

Figure 8. The process of the lateral compaction at: (a) The first time step; (b) The 33rd time step; (c) The 45th time step; (d) The 55th time step.

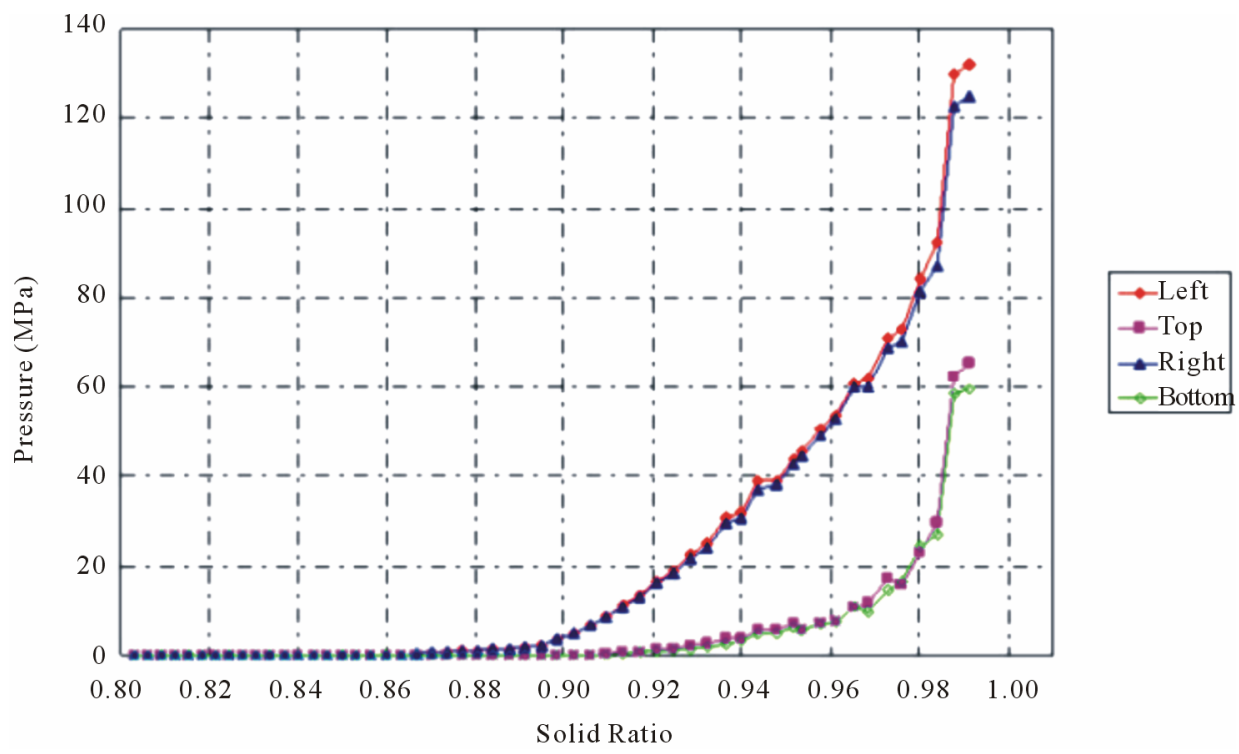

Figure 9. The relationship between the wall pressure and the solid ratio. 


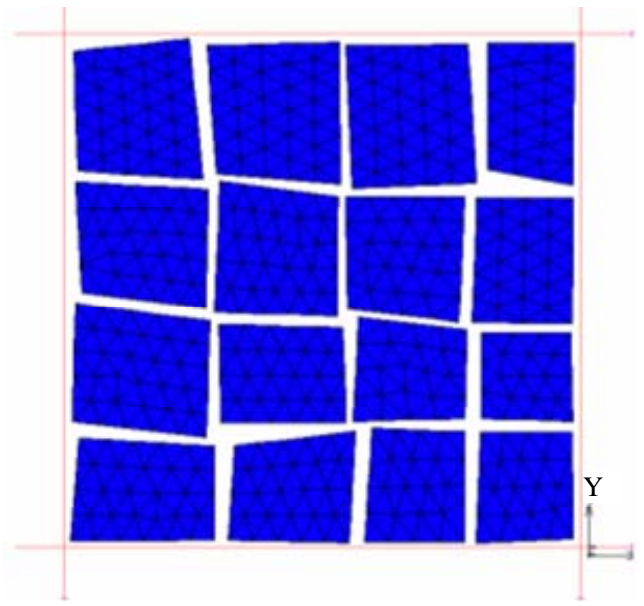

(a)

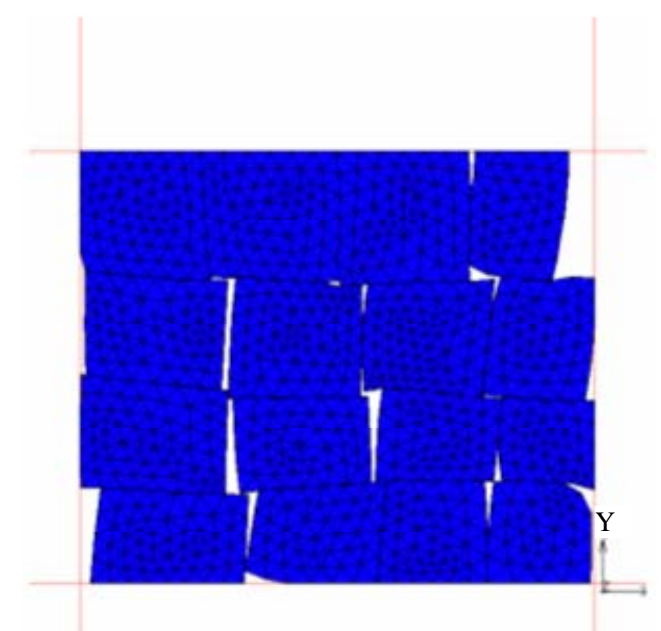

(c)

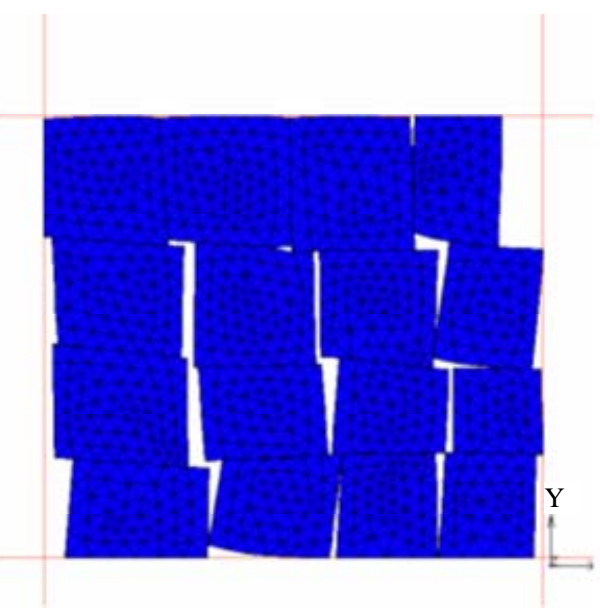

(b)

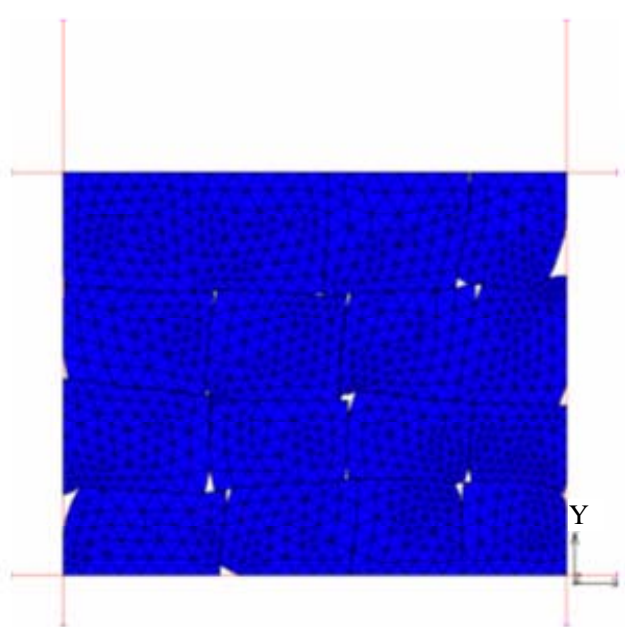

(d)

Figure 10. The process of the vertical compaction at: (a) The first time step; (b) The 32nd time step; (c) The 45th time step; (d) The 55th time step.

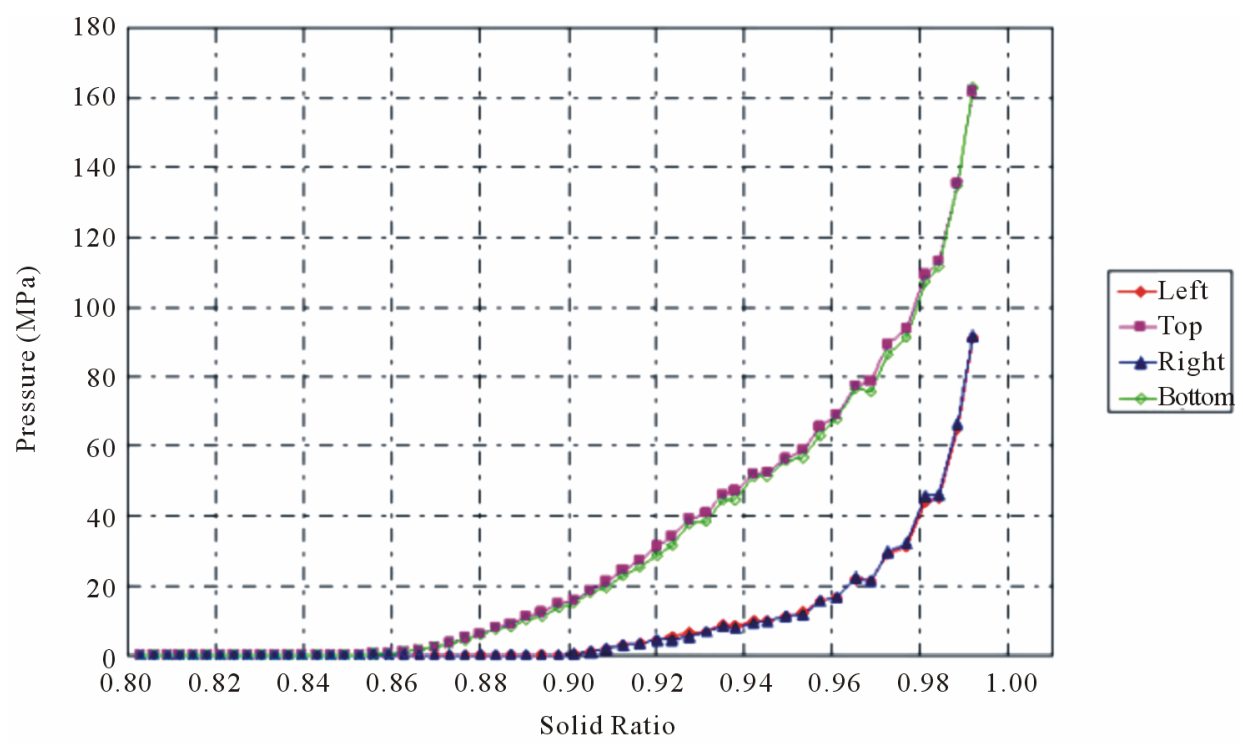

Figure 11. The relationship between the wall pressure and the solid ratio. 


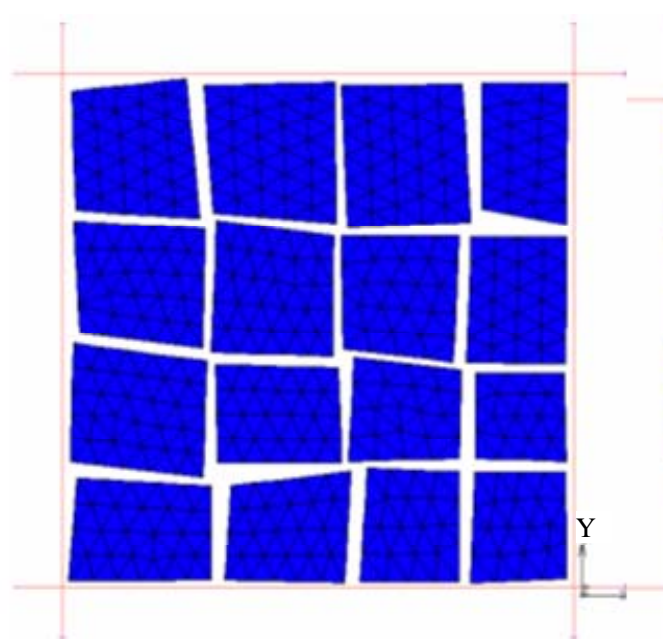

(a)

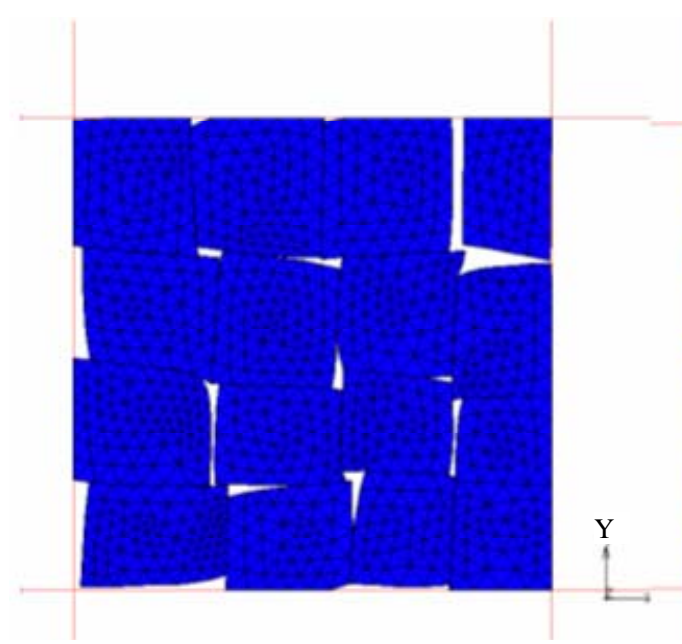

(c)

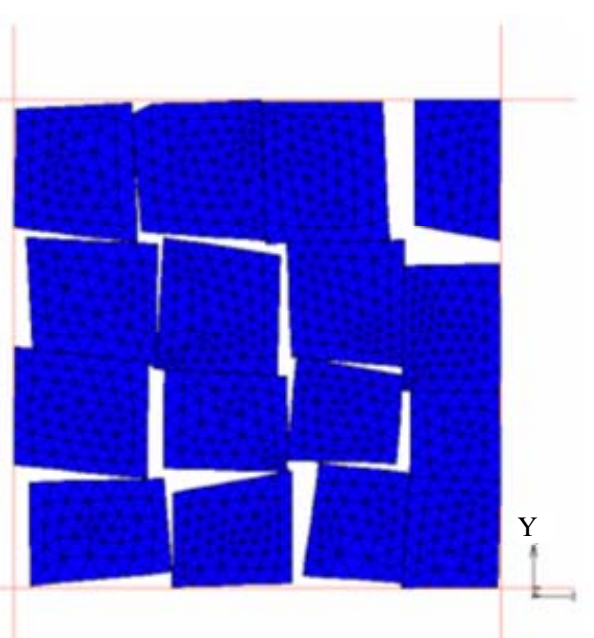

(b)

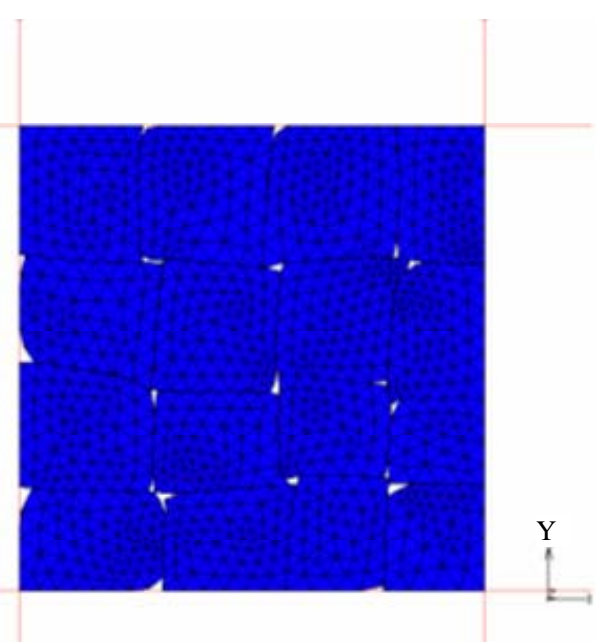

(d)

Figure 12. The process of the lateral compaction at: (a) The first time step; (b) The 22nd time step; (c) The 40th time step; (d) The 49th time step.

Figure 13 shows the relationship between the wall pressure and the solid ratio. The value of lateral pressure begins to increase when the solid ratio is about 0.86. A large increase in slope occurs when the solid ratio is close to 1 . When the solid ratio is $99.28 \%$, the values of the left wall pressure is $143.1 \mathrm{Mpa}$, the one of the right wall pressure is $146.1 \mathrm{MPa}$, the one of the top wall pressure is $132.9 \mathrm{MPa}$, and the one of the bottom wall pressure is $142.4 \mathrm{MPa}$. For the same solid ratio, the left-right pressure is less than the up-down pressure.

\subsection{The Mixed Visco-Plastic Analysis}

In this section, a series of mixed visco-plastic analyses for assembles of three types of asphalt are made to offer some reference data for asphalt industry. Three types of asphalt materials are used for analysis. Parameters of the material properties are shown in Table 3. Also Poisson ratio is set as 0.5 to ensure incompressibility of asphalt at high temperature and to provide the basis for error assessment.

Simulations made for compaction of 4 aggregates of asphalt have same area but are arranged in different ways shown in Figure 14. The simulation is regarded as a plane strain problem. Large deflection and large strain are taken into account in the process of the compaction. The large deflection and large strain operators are set in the calculation here. Meanwhile, to ensure the convergence of computation, all deformed meshes are generated adaptively. Friction between particles and wall is neglected. The inward movement of walls is of $10 \mathrm{~mm} / \mathrm{s}$ in velocity. Relationship of the wall pressure to solid ratio is obtained.

The relationships between pressure and solid ratio of 4 mixed aggregates are shown in Figure 15. It is indicated that, in descending order of required compaction pressure, the sequence is the first combination, the fourth combination, the third composition and second composition. 


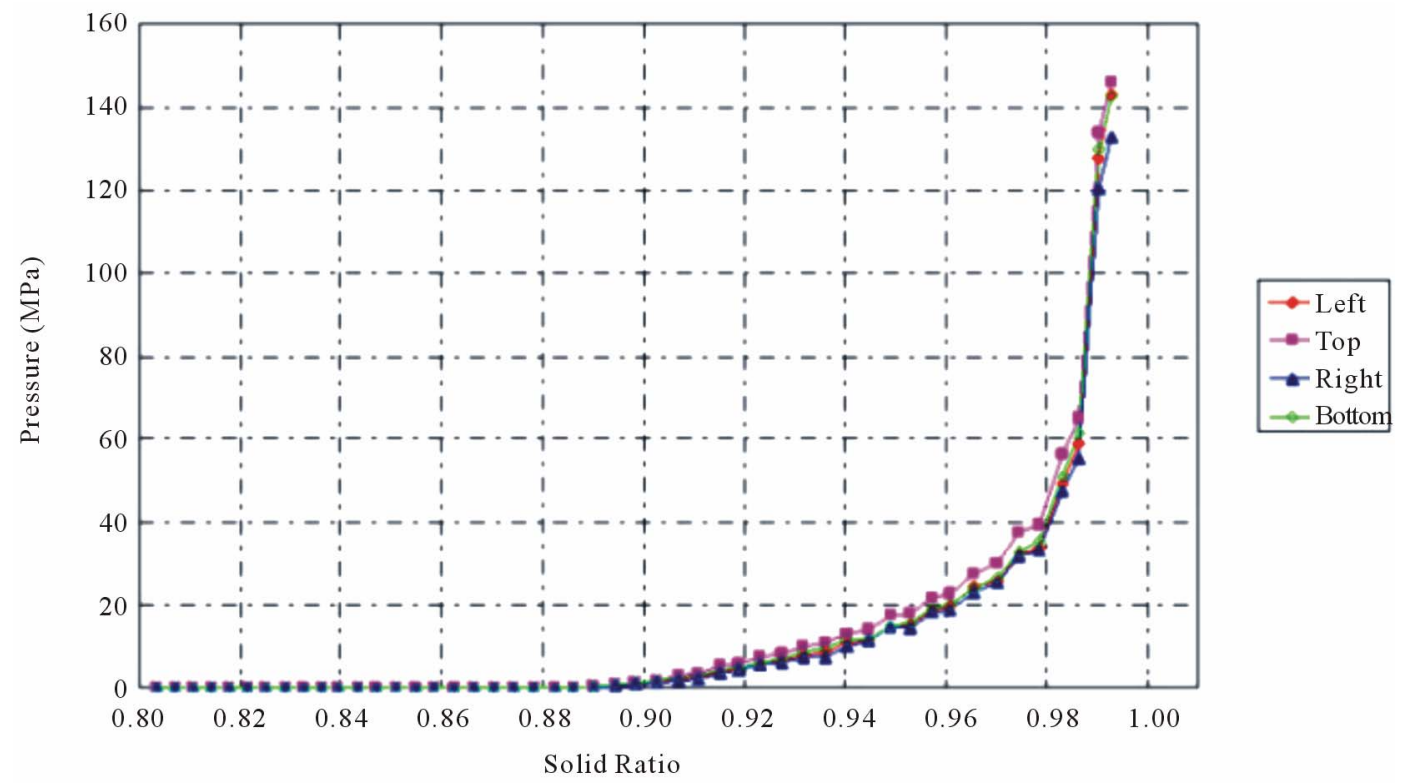

Figure 13. The relationship between the wall pressure and the solid ratio.

Table 3. Parameters of material properties of the three types of asphalts.

\begin{tabular}{cccccccc}
\hline & & \multicolumn{2}{c}{ Creep } & & \multicolumn{2}{c}{ Elastic } \\
\cline { 2 - 7 } Type & \multicolumn{1}{c}{$A$} & $n$ & $p$ & $q$ & $E / \mathrm{MPa}$ & $\mu$ \\
$\mathrm{nyyyyyy} \mathrm{m} 1$ & $5.15 \times 10^{-8}$ & 0 & 0.826 & 0 & 0.221 & 526 & 0.5 \\
$\mathrm{~m} 2$ & $1.41 \times 10^{-7}$ & 0 & 1.101 & 0 & 0.358 & 440 & 0.5 \\
$\mathrm{~m} 3$ & $6.28 \times 10^{-8}$ & 0 & 1.073 & 0 & 0.324 & 420 & 0.5 \\
\hline
\end{tabular}

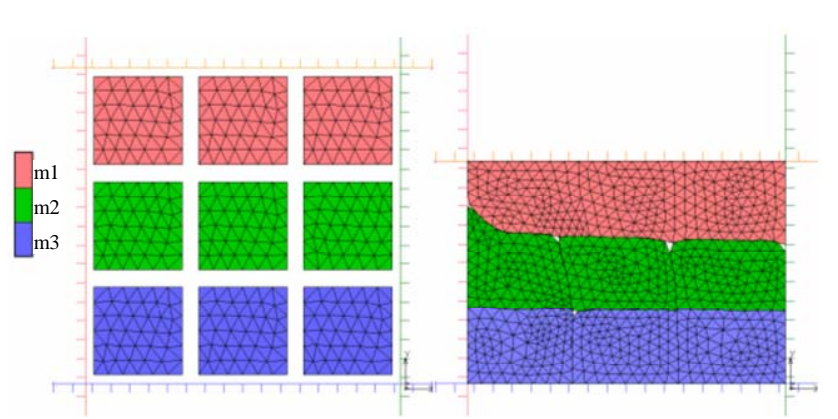

(a)

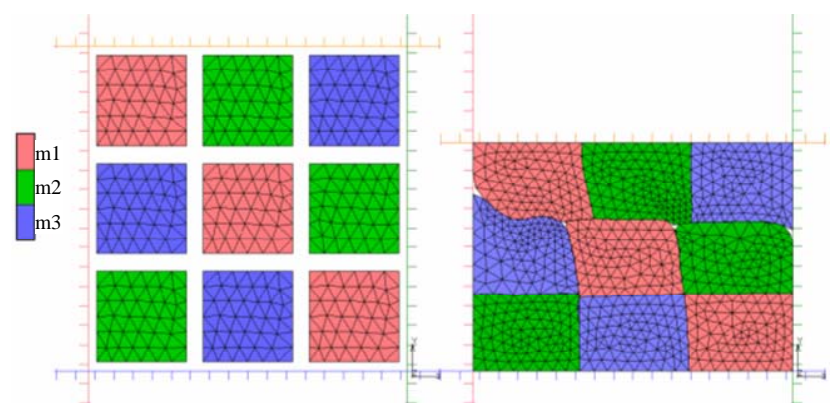

(c)

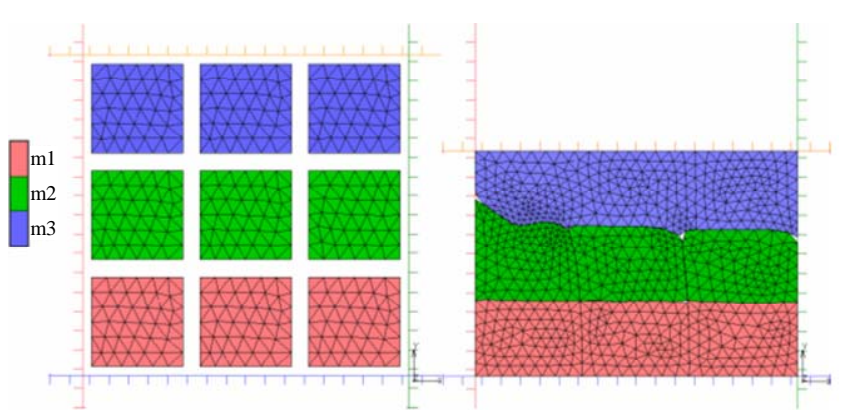

(b)

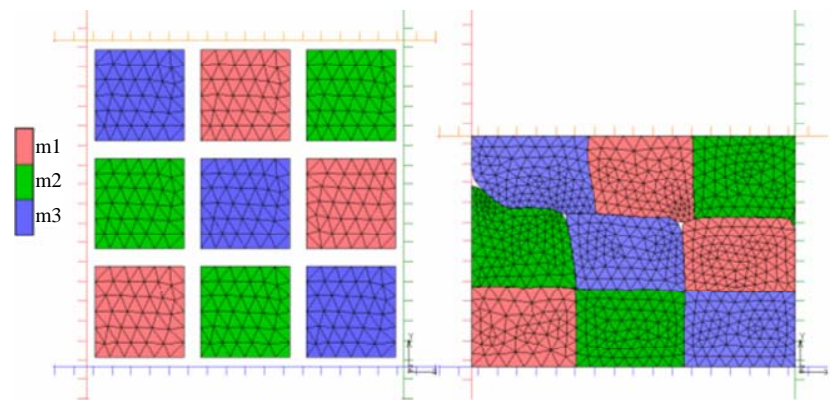

(d)

Figure 14. Vertical compaction of 4 assemblies of asphalt: their initial and final configurations. 


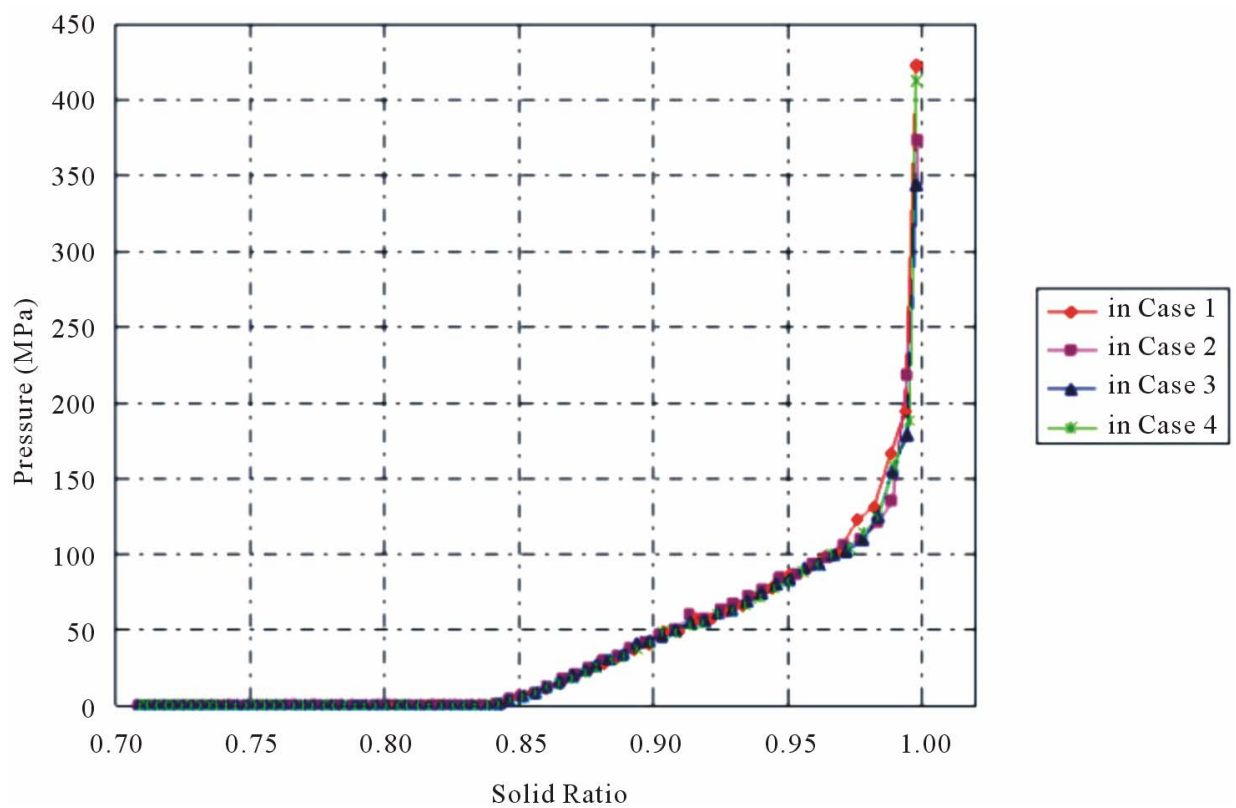

Figure 15. The relationships between pressure and solid ratio of 4 mixed aggregates.

The result shows reasonableness and superiority of actual road construction. It provides significant basis and reference for asphalt overlay industry.

\section{Results and Discussions}

\subsection{The Effect of Friction on the Compaction of the Elasto-Plastic Granular Assemblies}

The effect of friction on the compaction of the elastoplastic aggregates is investigated in this section. In Figure 16, a comparison among cases of 4 different contact conditions between particles, such as no friction, the friction factor is 0.1 , the friction factor is 0.2 , and sticking, is made. It is indicated that the condition without friction converges sooner than the other 3 cases. The reason might be that in the case of no friction gets less frictional energy loss. In general, the difference of pressure among these cases is approximately $20 \%$ or so. It is demonstrated that the effect of friction on the global behavior of the elasto-plastic granular assemblies under compacting is considerable.

\subsection{The Effect of the Initial Solid Volume Ratio on the Compaction of the Visco-Plastic Granular Assemblies}

The effect of the initial solid volume ratio on the global behavior of the visco-plastic granular assemblies under compacting is studied in this section. In Figure 17, a comparison among cases of 4 different initial solid volume ratios, such as $65 \%, 70 \%, 75 \%$, and $80 \%$, is made. It is shown that the case of the initial solid volume ratio as $65 \%$ converges sooner than the other 3 cases. The pres- sure difference among these 4 cases is about $15 \%$. It is demonstrated that the effect of the initial solid volume ratio is significant.

\subsection{The Influence of the Number of Particles in the Assembly on the Compaction of the Visco-Plastic Aggregates}

The influence of the number of particles in the assembly on the compaction of the visco-plastic aggregates is examined in this section. In Figure 18, a comparison among assemblies of 4 different numbers of particles, such as 1-particle assembly, 4-particle assembly, 9-particle assembly, and 16-particle assembly, is made. It is shown that the more particles the assembly has, the softer its material property is. The pressure difference among these 4 cases is approximately $10 \%$. It is demonstrated that the effect of the number of particles of the assembly on the global behavior of the visco-plastic aggregates is significant.

\subsection{The Comparison of Different Types of Compaction of the Elasto-Plastic Granular Assemblies}

The comparison of different types of compaction of the elasto-plastic granular assemblies, such as vertical compaction, horizontal compaction and biaxial compaction, is made, as shown in Figure 19. In general, the lateral pressure at the biaxial compaction is around 10\% higher than the one at the horizontal compaction, and is approximately $40 \%$ higher than the one at the vertical compaction. It is demonstrated that the difference among 


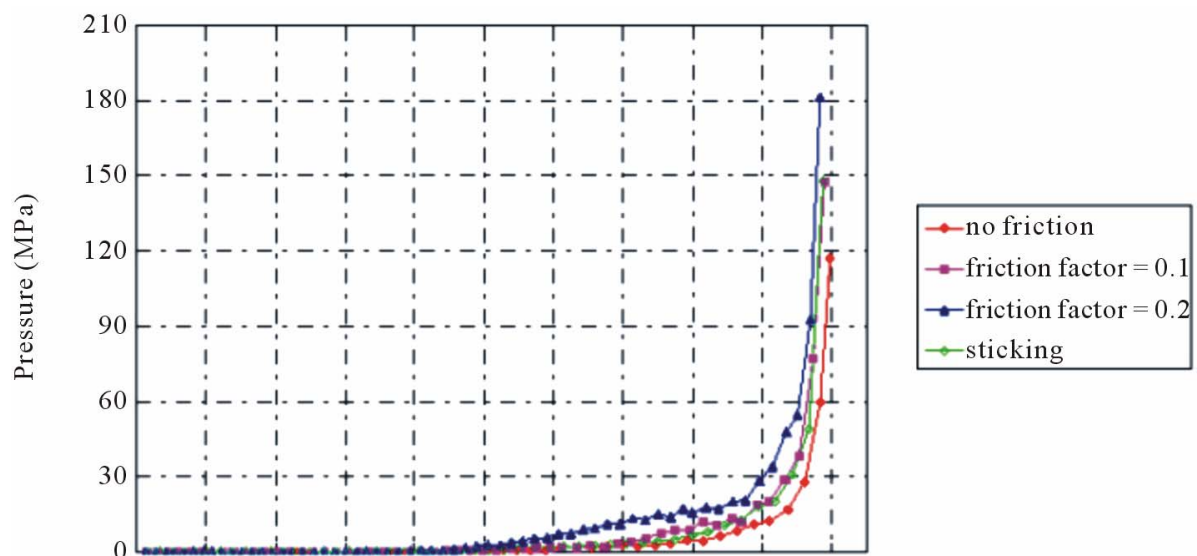

$\begin{array}{lllllllllll}0.80 & 0.82 & 0.84 & 0.86 & 0.88 & 0.90 & 0.92 & 0.94 & 0.96 & 0.98 & 1.00\end{array}$

Solid Ratio

Figure 16. A comparison among cases of 4 different contact conditions between particles is made.

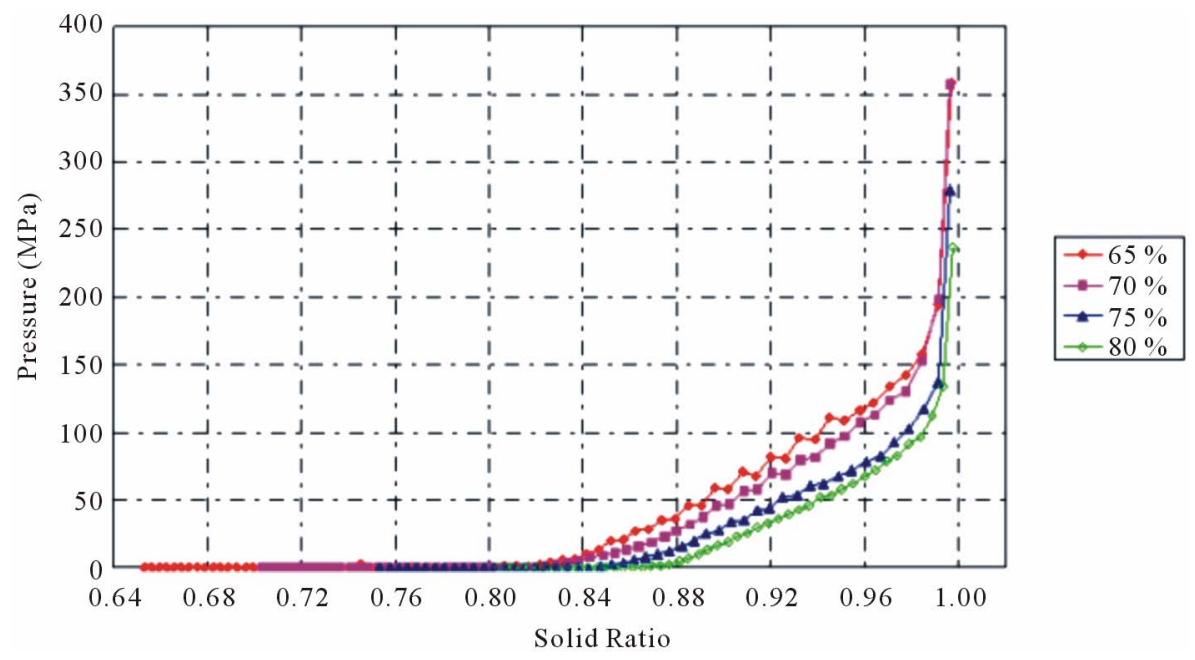

Figure 17. A comparison among cases of 4 different initial solid volume ratios, such as $65 \%, 70 \%, 75 \%$, and $80 \%$, is made.

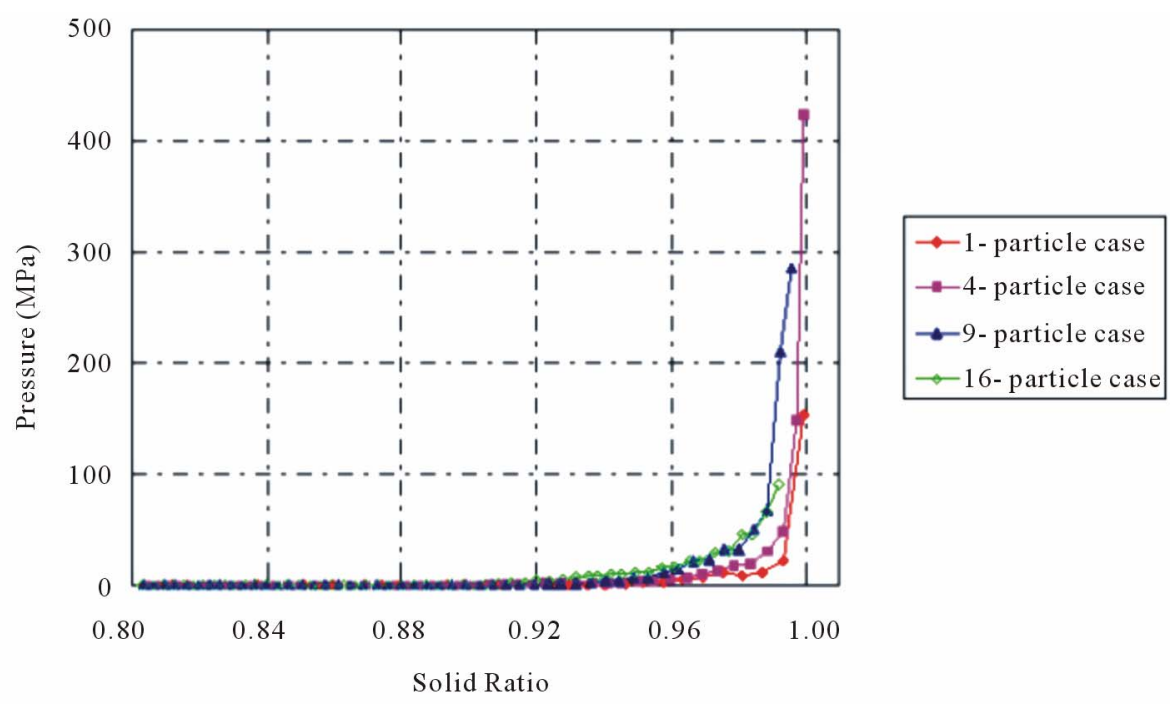

Figure 18. A comparison among assemblies of 4 different numbers of particles is made. 


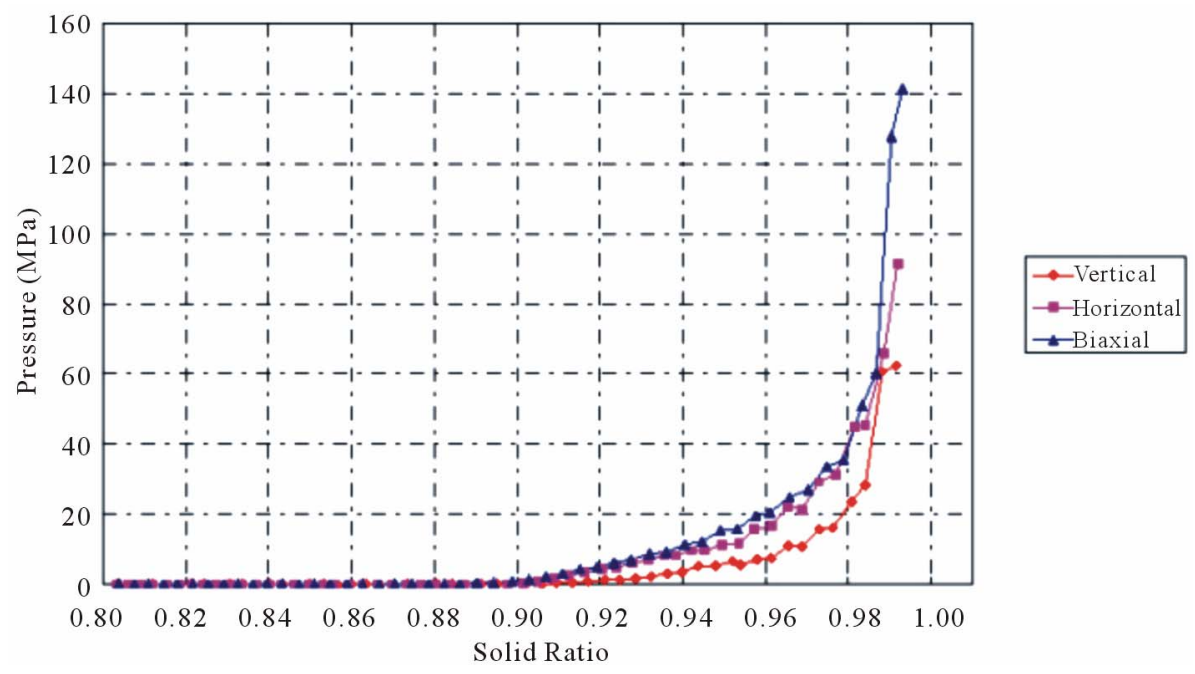

Figure 19. A comparison of different types of compaction, such as vertical compaction, horizontal compaction and biaxial compaction, is made.

different types of compaction is considerable.

\subsection{Error Estimation}

Error estimation is made for all simulations of compactions of the granular materials in this section. It is assumed that materials of the granular assemblies are incompressible. So one of the methods to estimate error of analytical results is calculating the ratio of the change of solid volume to the initial solid volume. In general, the average of errors in this study is about $0.6 \%$. It is demonstrated that the results of the numerical analyses are acceptable and satisfactory.

\section{Conclusions and Prospect}

In this paper, the compactions of elasto-plastic and viscoplastic polygonal granular materials are simulated using the finite element method. A couple of numerical experiments are made using the numerical model. Some new discoveries are presented as follows. First of all, in the simulation for the compaction of aggregates consising of particles of a single elasto-plastic incompressible material, it is demonstrated that if the initial solid volume ratios of the assemblies are the same, the more particles there are, the more oscillated the pressure-density curve is. But the general trend of the curve almost keeps the same. When the solid volume ratio is close to 1 , stress in the biaxial compaction is around 15\% lower than one in the uni-axial compaction. The effect of initial solid volume ratio as well as the effect of friction among particles on the stress-density relation is considerable. In addition, in the simulation for the compaction of aggregates consisting of particles of a single viscous incompressible material, if the initial volumes of the assemblies are the same, when the solid volume ratio is close to 1 , stress in the biaxial compaction is around $40 \%$ lower than one in the uni-axial compaction. Finally, the numerical model is extended to simulate the compaction of aggregates consisting of mixed particles of different viscous incompressible materials. It is indicated that, with minor modification, the method could be used in a variety of problems that can be represented using granular media, such as asphalt, polymers, aluminum, snow, food product, etc. However, the number of particles in the granular assemblies presented in this paper is less than 25. To extend the proposed numerical model to analyze the behavior of large-scale granular systems, more studies on multi-scale methods, statistical random sampling models and stochastic simulations are necessary.

\section{Acknowledgements}

This work is sponsored by National Natural Science Foundation of China under grant 10972162. This support is gratefully acknowledged.

\section{REFERENCES}

[1] H. M. Jaeger and S. R. Nagel, "The Physics of Granular Materials,” Physics Today, Vol. 49, No. 4, 1996, pp. 3238. doi:10.1063/1.881494

[2] S. Luding, "Stress Distribution in Static Two Dimensional Granular Model Media in the Absence of Friction," Physical Review E, Vol. 4, No. 55, 1997, pp. 4720-4729. doi:10.1103/PhysRevE.55.4720

[3] Y. M. Bashir and J. D. Goddard, "A Novel Simulation Method for the Quasi-Static Mechanics of Granular Assemblages," Journal of Rheology, Vol. 5, No. 35, 1991, pp. 849-885. doi:10.1122/1.550160

[4] M. A. Tzaferopoulos, "On the Numerical Modeling of Convex Particle Assemblies with Friction," Computer Methods in Applied Mechanics and Engineering, Vol. 127, 
No. 1-4, 1995, pp. 371-386. doi:10.1016/0045-7825(95)00852-8

[5] S. N. Coppersmith, C. H. Liu and O. Narayan, "Model for Force Fluctuations in Bead Packs," Physical Review E, Vol. 5, No. 53, 1996, pp. 4673-4685. doi:10.1103/PhysRevE.53.4673

[6] H. G. Matuttis, "Simulations of the Pressure Distribution under a Two Dimensional Heap of Polygonal Particles," Granular Matter, Vol. 2, No. 1, 1998, pp. 83-91. doi:10.1007/s100350050013

[7] M. Satake, "Tensorial form Definitions of Discrete-Mechanical Quantities for Granular Assemblies,” International Journal of Solids and Structures, Vol. 41, No. 21, 2004, pp. 5775-5791. doi:10.1016/j.ijsolstr.2004.05.046

[8] F. Emeriault and C. Claguin, "Statistical Homogenization for Assemblies of Elliptical Grains: Effect of the Aspect Ratio and Particle Orientation," International Journal of Solids and Structures, Vol. 41, No. 2, 2004, pp. 58375849. doi:10.1016/j.ijsolstr.2004.05.047
[9] S. J. Antony and R. Kuhn, "Influence of Particle Shape on Granular Contact Signatures and Shear Strength: New Insights from Simulations,” International Journal of Solids and Structures, Vol. 41, No. 21, 2004, pp. 5863-5870. doi:10.1016/j.ijsolstr.2004.05.067

[10] Y. C. Wu, E. G. Thompson, P. R. Heyliger and Z. H. Yao, "The Compaction of Blended Aggregates of Non-Spherical Linear Viscous Particles," Computer Methods in Applied Mechanics and Engineering, Vol. 193, No. 36-38, 2004, pp. 3871-3890. doi:10.1016/j.cma.2004.02.008

[11] Y. C. Wu, “Automatic Adaptive Mesh Upgrade Schemes of the Step-by-Step Incremental Simulation for Quasi Linear Viscoplastic Granular Materials,” Computer Methods in Applied Mechanics and Engineering, Vol. 197, 2008, pp. 1479-1494. doi:10.1016/j.cma.2007.11.020

[12] Y. Wu, J. Xiao and C. Zhu, "The Compaction of TimeDependent Viscous Granular Materials Considering Inertial Forces,” Acta Mechanica Solida Sinica, Vol. 24, No. 6, 2011, pp. 495-505. 